\title{
Front Matter: Volume 7753
}

, "Front Matter: Volume 7753," Proc. SPIE 7753, 21st International Conference on Optical Fiber Sensors, 775301 (17 May 2011); doi: $10.1117 / 12.898260$

SPIE Event: 21st International Conference on Optical Fibre Sensors (OFS21), SPIE. 2011, Ottawa, Canada 


\title{
PROCEEDINGS OF SPIE
}

\section{1st International Conference on Optical Fiber Sensors}

\author{
Wojtek J. Bock \\ Jacques Albert \\ Xiaoyi Bao \\ Editors

\section{5-19 May 2011} \\ Ottawa, Canada
}

Organized by

OCRI-Ottawa Centre for Research and Innovation (Canada)

CPC-Canadian Photonics Consortium (Canada)

Sponsored by

Oz Optics (Canada)

Simbol Test Systems, Inc. (Canada)

FISO Technologies, Inc. (Canada)

CMC Microsystems Corporation (Canada)

Innovative Economy: National Strategic Reference Framework (Poland)

Université du Québec en Outaouais (Canada)

IEEE Photonics Society

Photonics Research Center, Université du Québec en Outaouais (Canada)

Volume 7753

Part One of Two Parts

Proceedings of SPIE, 0277-786X, v. 7753 
The papers included in this volume were part of the technical conference cited on the cover and title page. Papers were selected and subject to review by the editors and conference program committee. Some conference presentations may not be available for publication. The papers published in these proceedings reflect the work and thoughts of the authors and are published herein as submitted. The publisher is not responsible for the validity of the information or for any outcomes resulting from reliance thereon.

Please use the following format to cite material from this book:

Author(s), "Title of Paper," in 21 st International Conference on Optical Fiber Sensors, edited by Wojtek J. Bock, Jacques Albert, Xiaoyi Bao, Proceedings of SPIE Vol. 7753 (SPIE, Bellingham, WA, 2011) Article CID Number.

ISSN 0277-786X

ISBN 9780819482464

Published by

SPIE

P.O. Box 10, Bellingham, Washington 98227-0010 USA

Telephone +1 3606763290 (Pacific Time) · Fax +1 3606471445

SPIE.org

Copyright (C) 2011, Society of Photo-Optical Instrumentation Engineers

Copying of material in this book for internal or personal use, or for the internal or personal use of specific clients, beyond the fair use provisions granted by the U.S. Copyright Law is authorized by SPIE subject to payment of copying fees. The Transactional Reporting Service base fee for this volume is $\$ 18.00$ per article (or portion thereof), which should be paid directly to the Copyright Clearance Center (CCC), 222 Rosewood Drive, Danvers, MA 01923. Payment may also be made electronically through CCC Online at copyright.com. Other copying for republication, resale, advertising or promotion, or any form of systematic or multiple reproduction of any material in this book is prohibited except with permission in writing from the publisher. The CCC fee code is 0277-786X/11/ \$18.00.

Printed in the United States of America.

Publication of record for individual papers is online in the SPIE Digital Library.

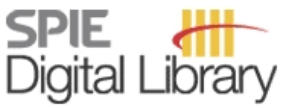

SPIEDigitalLibrary.org

Paper Numbering: Proceedings of SPIE follow an e-First publication model, with papers published first online and then in print and on CD-ROM. Papers are published as they are submitted and meet publication criteria. A unique, consistent, permanent citation identifier (CID) number is assigned to each article at the time of the first publication. Utilization of CIDs allows articles to be fully citable as soon they are published online, and connects the same identifier to all online, print, and electronic versions of the publication. SPIE uses a six-digit CID article numbering system in which:

- The first four digits correspond to the SPIE volume number.

- The last two digits indicate publication order within the volume using a Base 36 numbering system employing both numerals and letters. These two-number sets start with 00, 01, 02, 03, 04, $05,06,07,08,09,0 A, 0 B \ldots 0 Z$, followed by 10-1Z, 20-2Z, etc.

The CID number appears on each page of the manuscript. The complete citation is used on the first page, and an abbreviated version on subsequent pages. Numbers in the index correspond to the last two digits of the six-digit CID number. 


\section{Contents}

xxxvii Conference Committee

xli Introduction

\section{A LOOK BACK AT OPTICAL FIBER SENSORS}

775302 Optical fiber sensor research and industry in Germany: review and outlook (Invited Paper) [7753-475]

R. Willsch, W. Ecke, H. Bartelt, Institut für Photonische Technologien e.V. (Germany)

775303 The origin, history and future of fiber optic interferometric acoustic sensors for US Navy applications (Invited Paper) [7753-466]

J. H. Cole, J. A. Bucaro, C. K. Kirkendall, A. Dandridge, U.S. Naval Research Lab. (United States)

\section{CURRENT FIBER SENSOR APPLICATIONS AND TECHNOLOGIES}

775304 Pressure measurement with fiber optic sensors: commercial technologies and applications (Invited Paper) [7753-500]

É. Pinet, FISO Technologies, Inc. (Canada)

775305 Distributed sensing with OFDR and its application to structural health monitoring (Invited Paper) [7753-382]

H. Murayama, The Univ. of Tokyo (Japan); H. Igawa, Japan Aerospace Exploration Agency (Japan); K. Omichi, Fujikura Ltd. (Japan); Y. Machijima, Lazoc Inc. (Japan)

\section{PLENARY TALK}

775306 Sensing high speed phenomena using fiber gratings and the Sagnac interferometer (Plenary Paper) [7753-105]

E. Udd, Columbia Gorge Research (United States)

\section{BRILLOUIN SENSORS}

775307 Brillouin scattering accompanied by acoustic grating in an optical fiber and applications in fiber distributed sensing (Invited Paper) [7753-477]

K. Hotate, The Univ. of Tokyo (Japan)

$775308 \quad 100 \mathrm{~km}$ sensing range Brillouin optical time domain analysis based on time-division multiplexing [7753-265]

Y. Dong, X. Bao, L. Chen, Univ. of Ottawa (Canada) 
775309 Hot spot detection over 100 km with 2 meter resolution in a Raman-assisted Brillouin distributed sensor [7753-182]

X. Angulo-Vinuesa, S. Martin-Lopez, J. Nuño, P. Corredera, J. D. Ania-Castañon, Consejo Superior de Investigaciones Cientificas (Spain); L. Thévenaz, Ecole Polytechnique Fédérale de Lausanne (Switzerland); M. Gonzalez-Herraez, Univ. de Alcalá (Spain)

7753 OA Measurement range elongation based on a temporal gating scheme in Brillouin correlation domain distributed discrimination system for strain and temperature operated by a single laser [7753-203]

R. K. Yamashita, The Univ. of Tokyo (Japan); W. Zou, Shanghai Jiao Tong Univ. (China); Z. He, K. Hotate, The Univ. of Tokyo (Japan)

7753 OB Faraday rotating Brillouin sensor system [7753-178]

A. W. Brown, Dark-Pulse Technologies Ltd. (Canada); M. T. V. Wylie, B. G. Colpitts, Univ. of New Brunswick (Canada)

MICRO- AND NANO-FIBERS

7753 OC Optical fibre microwire sensors (Invited Paper) [7753-14]

G. Brambilla, M. Belal, Y. Jung, Z. Song, F. Xu, T. Newson, D. Richardson, Univ. of Southampton (United Kingdom)

7753 OD Self-assembly and nanotechnology within an optical fibre for improved evanescent field sensing [7753-301]

J. Canning, W. Padden, D. Boskovic, M. Naqshbandi, L. Costanza, H. de Bruyn, H. T. Sum, M. J. Crossley, The Univ. of Sydney (Australia)

7753 OE Tunable Fabry-Perot filter based on hollow-core photonic bandgap fiber and micro-fiber and its application [7753-462]

X. Wang, Univ. of Ottawa (Canada); T. Zhu, Univ. of Ottawa (Canada) and Chongaing Univ. (China); L. Chen, X. Bao, Univ. of Ottawa (Canada)

7753 OF An optical trapping based microfiber vibration sensor [7753-355]

L. Wang, P. Liang, Z. Liu, A. Zhou, L. Yuan, Harbin Engineering Univ. (China)

7753 OG Etched core fiber Bragg grating sensor integrated with microfluidic channel [7753-451] S.-M. Lee, M.-Y. Jeong, Pusan National Univ. (Korea, Republic of); S. S. Saini, Univ. of Waterloo (Canada)

\section{SENSOR APPLICATIONS}

$7753 \mathrm{OH} \quad$ Demodulation technique for plasmonic fiber grating sensors using orthogonally polarized light states [7753-354]

C. Caucheteur, Carleton Univ. (Canada) and Univ. de Mons (Belgium); Y. Y. Shevchenko, L. Shao, Carleton Univ. (Canada); P. Mégret, Univ. de Mons (Belgium); J. Albert, Carleton Univ. (Canada) 
7753 Ol In situ radiation influence on strain measurement performance of Brillouin sensors [7753-359] $X$. Pheron, ANDRA (France) and Lab. Hubert Curien, CNRS (France); Y. Ouerdane, Lab. Hubert Curien, CNRS (France); S. Girard, C. Marcandella, CEA DAM (France); S. Delepine-Lesoille, J. Bertrand, ANDRA (France); F. Taillade, E. Merliot, Lab. Central des Ponts et Chaussées (France); Y. Sikali Mamdem, EDF Recherche \& Developpement (France); A. Boukenter, Lab. Hubert Curien, CNRS (France)

$77530 \mathrm{~J}$ Self-packaged Type II femtosecond IR laser induced fiber Bragg grating for temperature applications up to $1000^{\circ} \mathrm{C}$ [7753-456]

D. Grobnic, S. J. Mihailov, R. B. Walker, C. W. Smelser, Communications Research Ctr. Canada (Canada)

7753 OK Evaluation of the transversal strain state in a satin weave composite using fibre Bragg gratings [7753-253]

E. Voet, G. Luyckx, J. Degrieck, W. Van Paepegem, Univ. Gent (Belgium)

POSTER SESSION: MACH-ZEHNDER, SPR, FABRY-PEROT, INTERFEROMETER, RESONATOR, BRILLOUIN, DISTRIBUTED, LASER

$7753 \mathrm{OL} \quad$ A novel positioning method for dual Mach-Zehnder interferometric vibration sensor in submarine cable security application [7753-255]

S. Xie, M. Zhang, Y. Li, Y. Liao, Tsinghua Univ. (China)

7753 OM Fiber in-line Mach-Zehnder interferometer based on selective infiltration of photonic crystal fiber [7753-276]

M. Yang, D. N. Wang, Y. Wang, C. R. Liao, The Hong Kong Polytechinic Univ. (Hong Kong, China)

7753 ON In-line all-fiber Fabry-Perot and Mach-Zehnder interferometers formed by hollow fiber with lateral offset [7753-92]

Y.-J. Rao, Chongqing Univ. (China) and Univ. of Electronic Science \& Technology of China (China); D. W. Duan, L. C. XU, M. Deng, T. Zhu, Chongqing Univ. (China)

$775300 \quad$ Experimental demonstration on $2.5 \mathrm{~m}$ spatial resolution and $1^{\circ} \mathrm{C}$ temperature uncertainty over 74.6km BOTDA with combined Raman amplification and optical pulse coding [7753-101]

Y.-J. Rao, X.-H. Jia, K. Deng, Z.-X. Yang, L. Chang, C. Zhang, Z.-L. Ran, Univ. of Electronic Science and Technology of China (China)

7753 OP A novel quasi-distributed sensing network based on non-balance Mach-Zehnder autocorrelator [7753-285]

J. Yang, Y. Yuan, A. Zhou, L. Yuan, Harbin Engineering Univ. (China)

$77530 Q \quad$ Refractive index and temperature sensor based on double-pass in-line Mach-Zehnder interferometer [7753-122]

Y. Li, China Jiliang Univ. (China) and Univ. of Ottawa (Canada); L. Chen, E. Harris, X. Bao, Univ. of Ottawa (Canada); W. J. Bock, Univ. du Québec en Outaouais (Canada) 
7753 OR Fiber loop ringdown strain sensor with photonic crystal fiber based Mach-Zehnder interferometer [7753-165]

W. Zhou, China Jiliang Univ. (China) and Nanyang Technological Univ. (Singapore);

W. C. Wong, C. C. Chan, Nanyang Technological Univ. (Singapore); L.-Y. Shao, China Jiliang Univ. (China) and Carleton Univ. (Canada); X. Dong, China Jiliang Univ. (China)

7753 OS Curvature measurement with photonic crystal fiber based Mach-Zehnder interferometer [7753-97]

M. Deng, C.-P. Tang, T. Zhu, Chongqing Univ. (China); Y.-J. Rao, Univ. of Electronic Science and Technology of China (China) and Chongqing Univ. (China)

7753 OT PCF-based Fabry-Perot interferometric sensor for strain measurement under high-temperature [7753-93]

M. Deng, C.-P. Tang, T. Zhu, Chongqing Univ. (China); Y.-J. Rao, Univ. of Electronic Science and Technology of China (China) and Chongqing Univ. (China)

7753 OU Microstructured fiber Mach-Zehnder interferometers for simultaneous measurement of axial strain and temperature [7753-409]

Q. Chen, P. LU, Memorial Univ. of Newfoundland (Canada)

7753 OV Polarization-dependent in-line Mach-Zehnder interferometer for discrimination of temperature and ambient index [7753-89]

H.-J. Kim, Y. Y.-G. Han, Hanyang Univ. (Korea, Republic of)

7753 OW Miniaturized fiber optic surface-plasmon-resonance sensor [7753-474]

T. Schuster, N. Neumann, Technische Univ. Dresden (Germany); C. Schäffer,

Helmut-Schmidt Univ. (Germany)

7753 OX Improved performance of SPR optical fiber sensors with InN as dielectric cover [7753-207]

Ó. Esteban, F. B. Naranjo, Univ. de Alcalá de Henares (Spain); N. Díaz-Herrera, Univ. Complutense de Madrid (Spain); S. Valdueza-Felip, Univ. de Alcalá de Henares (Spain);

M. C. Navarrete, A. González Cano, Univ. Complutense de Madrid (Spain)

7753 OY An experimental evaluation of the behavior of SPR fiber sensors in absorptive medium when plasmons are tuned to absorption peaks: method for selective measurement [7753-297]

I. Leite, Univ. de Alcalá de Henares (Spain); M. C. Navarrete, N. Díaz-Herrera, A. Gonzalez Cano, Univ. Complutense de Madrid (Spain); O. Esteban, Univ. de Alcalá de Henares (Spain)

$77530 Z$ Chemical sensing with an all-fiber reflection LSPR sensor [7753-368]

P. M. P. Gouvêa, D. P. Parra, A. M. B. Braga, I. C. S. Carvalho, Pontifícia Univ. Católica do Rio de Janeiro (Brazil)

775310 Surface plasmon resonance refractive index fiber sensor with hole-assisted structure [7753-107]

C. Guan, L. Yuan, Harbin Engineering Univ. (China)

775311 Development and sensitivity studies of a gold nanorod platform for a localized surface plasmon resonance based optical fibre biosensor [7753-325]

J. Cao, The City Univ. (United Kingdom) and Harbin Institute of Technology (China);

E. Galbraith, T. Sun, K. T. V. Grattan, The City Univ. (United Kingdom) 
775312 A novel fiber-tip micro-cavity sensor for high temperature application [7753-385] J. Ma, W. Jin, L. Jin, The Hong Kong Polytechnic Univ. (Hong Kong, China)

775313 Porous silicon-based optical fiber Fabry-Perot sensor for relative humidity determination [7753-96] G. Rong, S. Pan, C. WU, Shanghai Jiao Tong Univ. (China); X. Li, M. Yang, Wuhan Univ. of Technology (China)

775314 Inspection technique for cleaved optical fiber ends based on Fabry-Perot resonator [7753-215]

M. Kihara, H. Watanabe, Y. Yajima, M. Toyonaga, Nippon Telegraph and Telephone East Corp. (Japan)

775315 Fiber optic Fabry-Perot sensor based on graded-index multimode fiber: numerical simulations and experiments [7753-100]

Y. Gong, T. Zhao, Y.-J. Rao, Y. Wu, Y. Guo, Univ. of Electronic Science and Technology of China (China)

775316 Demodulation of micro fiber optic Fabry-Perot interferometer using subcarrier and dual-wavelength method [7753-109]

Z. Ran, Y. Rao, Z. Liu, F. Xu, Univ. of Electronic Science and Technology of China (China)

$775317 \quad 1100^{\circ} \mathrm{C}$ fiber optic high-temperature Fabry-Perot sensors fabricated by laser-micromachining [7753-113]

Z. Ran, Y. Chen, Y. Rao, D. Sun, E. Lu, Z. Liu, Univ. of Electronic Science and Technology of China (China)

775318 Fiber optic Fabry-Perot interferometer tip accelerometer fabricated by laser-micromachining [7753-114]

Z. Ran, E. Lu, Y. Rao, M. Ni, F. Peng, D. Zeng, Univ. of Electronic Science and Technology of China (China)

775319 Fiber Fabry-Perot interferometer sensor for measuring resonances of piezoelectric elements [7753-299]

R. E. da Silva, R. A. Oliveira, A. A. P. Pohl, Federal Univ. of Technology (Brazil)

$77531 \mathrm{~A}$ Temperature Raman laser sensor based in a suspended-core Fabry-Perot cavity and cooperative Rayleigh scattering [7753-281]

A. M. R. Pinto, M. Lopez-Amo, Univ. Pública de Navarra (Spain); J. Kobelke, K. Schuster, Institute of Photonic Technology (Germany)

7753 1B High-sensitivity salinity sensor realized with photonic crystal fiber Sagnac interferometer [7753-376]

C. Wu, The Hong Kong Polytechnic Univ. (Hong Kong, China) and Dalian Univ. of Technology (China); H. Y. Fu, H. Y. Au, The Hong Kong Polytechnic Univ. (Hong Kong, China); B. O. Guan, Dalian Univ. of Technology (China) and Jinan Univ. (China); H. Y. Tam, The Hong Kong Polytechnic Univ. (Hong Kong, China)

7753 1C A hybrid Sagnac interferometer for discrimination of ambient index and temperature [7753-28]

O.-J. Kwon, Y. B. Shim, Y.-G. Han, Hanyang Univ. (Korea, Republic of) 
7753 1D Long distance simultaneous measurement of bending and temperature based on a dual-wavelength Raman fiber laser [7753-46]

O.-J. Kwon, H.-J. Kim, M.-S. Yoon, S. Park, Y. Shim, S. B. Lee, Y.-G. Han, Hanyang Univ. (Korea, Republic of)

7753 1E A Sagnac loop sensor for simultaneous strain and temperature measurement [7753-279] J. Kang, China Jiliang Univ. (China) and Univ. of Shanghai for Science and Technology (China); X. Dong, C. Zhao, China Jiliang Univ. (China); Y. Zhao, Zhejiang Tianma Bearing Co., Ltd (China); M. Li, Univ. of Shanghai for Science and Technology (China)

7753 IF Interferometric phase sensor using single-ended polarization maintaining fiber in Sagnac interferometer [7753-350]

Y. R. Yoon, M. H. Seo, H. D. Lee, M. Y. Jeong, C.-S. Kim, Pusan National Univ. (Korea, Republic of)

$77531 \mathrm{G} \quad$ A novel magnetic field fiber sensor by using magnetic fluid in Sagnac loop [7753-44] P. Zu, C. C. Chan, Nanyang Technological Univ. (Singapore); Y. Jin, China Jiliang Univ. (China); Y. Zhang, Nanyang Technological Univ. (Singapore); X. Dong, China Jiliang Univ. (China)

$7753 \mathrm{lH} \quad$ Resonator micro optic gyro with double phase modulation technique using an FPGA-based digital processor [7753-277]

H. Ma, H. Mao, Y. Chen, Z. Jin, Zhejiang Univ. (China)

$775311 \quad$ Optical-frequency-comb based interrogation of fiber resonators [7753-317]

G. Gagliardi, S. Avino, Istituto Nazionale di Ottica, CNR (Italy); M. Fabian, Univ. of Limerick (Ireland); M. Salza, P. Ferraro, P. De Natale, Istituto Nazionale di Ottica, CNR (Italy)

7753 1 J A novel optical fiber current sensor using polarization diversity and a Faraday rotation mirror cavity [7753-273]

H. Zhang, Y. Dong, J. Leeson, L. Chen, X. Bao, Univ. of Ottawa (Canada)

$77531 \mathrm{~K}$ Offset errors caused by the resonance asymmetry in the waveguide-type optical passive resonator gyro [7753-163]

Y. Chen, H. Ma, Z. Jin, Zhejiang Univ. (China)

$77531 \mathrm{~L} \quad$ Microscopic multiple-point temperature sensing based on microfiber double-knot resonators [7753-169]

Y. Wu, Y. Chen, Y. Rao, T. Zhang, Y. Gong, Univ. of Electronic Science and Technology of China (China)

$77531 \mathrm{M}$ Interferometric humidity sensors based on microfiber knot resonators [7753-1 10] Y. Wu, T. Zhang, Y. Rao, Y. Gong, Univ. of Electronic Science and Technology of China (China)

7753 iN Dual-probe simultaneous measurements of refractive index and thickness with spectral-domain low coherence interferometry [7753-256]

S. J. Park, K. S. park, Y. H. Kim, Gwangju Institute of Science and Technology (Korea, Republic of); S.-J. Baik, Chonnam National Univ. (Korea, Republic of); B. H. Lee, Gwangju Institute of Science and Technology (Korea, Republic of) 
775310 Analysis of optical fiber interferometer sensor bonded to flat diaphragm for dynamic stress measurement at high temperature [7753-461]

H. Krisch, Krohne Messtechnik GmbH \& Co. KG (Germany); T. Nasilowski, Military Univ. of Technology (Poland); N. Fernandes, Krohne Marshall Pvt Ltd. (India); M. Lau,

P. Lutkowski, M. Skupski, K. Gossner, S. Tournillon, Krohne Messtechnik GmbH \& Co. KG (Germany); R. Plaga, Warsaw Univ. of Technology (Poland); L. Jaroszewicz, Military Univ. of Technology (Poland); T. Wolinski, Warsaw Univ. of Technology (Poland)

$77531 \mathrm{P} \quad$ Photonic crystal fiber interferometer for dew detection [7753-241]

J. Mathew, Y. Semenova, G. Rajan, G. Farrell, Dublin Institute of Technology (Ireland)

$77531 Q \quad$ Miniaturized fiber probe reflective interferometer sensor [7753-314]

J. Kou, J. Feng, F. Xu, Y. Lu, Nanjing Univ. (China)

7753 IR Inline core-cladding intermodal interferometer based on nano-coated photonic crystal fiber for refractive-index sensing [7753-326]

M. Smietana, Univ. du Québec en Outaouais (Canada) and Warsaw Univ. of Technology (Poland); D. Brabant, W. J. Bock, P. Mikulic, Univ. du Québec en Outaouais (Canada);

T. Eftimov, The Paissi Hilendarski Univ. of Plovdiv (Bulgaria)

7753 is All-fiber interferometric sensor of $150 \mathrm{kHz}$ acoustic emission for the detection of partial discharges within power transformers [7753-278]

J. E. Posada, J. Rubio-Serrano, J. A. Garcia-Souto, Univ. Carlos III de Madrid (Spain)

7753 1T Delayed self-heterodyne interferometry using Faraday mirrors in a Michelson configuration [7753-302]

M. L. Åslund, A. Canagasabey, A. Michie, J. Canning, The Univ. of Sydney (Australia);

J. Holdsworth, Univ. of Newcastle (Australia); S. Fleming, The Univ. of Sydney (Australia)

$77531 \mathrm{U}$ Interferometric system controlled by virtual instrumentation for differential thermal analysis [7753-374]

L. C. Gonçalves, INESC Porto (Portugal) and Univ. da Madeira (Portugal);

G. González-Aguilar, INESC Porto (Portugal); J. M. Baptista, INESC Porto (Portugal) and Univ. da Madeira (Portugal); P. A. S. Jorge, INESC Porto (Portugal)

$77531 \mathrm{~V}$ Tactical-grade interferometric fiber optic gyroscope driven with a narrow-linewidth laser [7753-379]

S. W. Lloyd, M. J. F. Digonnet, S. Fan, Stanford Univ. (United States)

7753 IW A simplified common-path autocorrelator based on Fizeau interferometer [7753-286]

Y. Yuan, J. Yang, A. Zhou, L. Yuan, Harbin Engineering Univ. (China)

$77531 \mathrm{X}$ Dynamical interrogation of interferometric sensor arrays by a simple polarimetric setup [7753-412]

Y. Katz, A. Eyal, Tel-Aviv Univ. (Israel)

$77531 \mathrm{Y} \quad$ Compact photonic crystal fiber refractometer based on modal interference [7753-61] W. C. Wong, C. C. Chan, Z. Q. Tou, L. H. Chen, Nanyang Technological Univ. (Singapore); K. C. Leong, GLOBALFOUNDRIES Inc. (Singapore) 
775312 Self-mixing interference in fiber ring laser and its multiplexing [7753-66] M. Wang, W. Xia, X. Dai, Y. Zhao, Nanjing Normal Univ. (China)

775320 Matrix operators for complex interferometer analysis [7753-443]

R. P. Dahlgren, Univ. of California, Santa Cruz (United States) and The SETI Institute (United States)

775321 Simplified Brillouin optical time-domain sensor based on direct modulation of a laser diode [7753-291]

K.-Y. Song, S. Yang, Chung-Ang Univ. (Korea, Republic of)

775322 Impact of pump depletion on the determination of the Brillouin gain frequency in distributed fiber sensors [7753-210]

L. Thévenaz, S. Foaleng Mafang, J. Lin, Ecole Polytechnique Fédérale de Lausanne (Switzerland)

775323 Distributed and dynamical Brillouin sensing in optical fibers [7753-289]

Y. Peled, A. Motil, L. Yaron, M. Tur, Tel-Aviv Univ. (Israel)

775324 Long integral temperature Brillouin sensor for off- shore wind energy power supply lines [7753-308]

M. A. Quintela, A. Ullán, A. Quintela, C. Galindez, Univ. de Cantabria (Spain);

R. A. Perez-Herrera, M. López-Amo, Univ. Pública de Navarra (Spain); J. M. Lopez-Higuera, Univ. de Cantabria (Spain)

775325 BOTDA sensor with 2-m spatial resolution over $120 \mathrm{~km}$ distance using bi-directional distributed Raman amplification [7753-162]

M. A. Soto, S. Faralli, M. Taki, G. Bolognini, F. Di Pasquale, Scuola Superiore Sant'Anna (Italy)

775326 Experimental examination of the variation of the spontaneous Brillouin power and frequency shift under the combined influence of temperature and strain [7753-357] M. Belal, T. P. Newson, Univ. of Southampton (United Kingdom)

775327 Evaluation of a high spatial resolution temperature compensated distributed strain sensor using a temperature controlled strain rig [7753-366]

M. Belal, T. P. Newson, Univ. of Southampton (United Kingdom)

775328 Simultaneous temperature and strain measurement with bandwidth and peak of the Brillouin spectrum in LEAF fiber [7753-471]

X. Liu, X. Bao, Univ. of Ottawa (Canada)

775329 Potential of Brillouin scattering in polymer optical fiber for strain-insensitive high-accuracy temperature sensing [7753-07]

Y. Mizuno, K. Nakamura, Tokyo Institute of Technology (Japan)

7753 2A Expansion of spatial measurement range in a correlation based Brillouin optical sensing system [7753-36]

J. H. Jeong, Korea Institute of Science and Technology (Korea, Republic of) and Hanyang Univ. (Korea, Republic of); K. Lee, Korea Institute of Science and Technology (Korea,

Republic of); J.-M. Jeong, Hanyang Univ. (Korea, Republic of); S. B. Lee, Korea Institute of Science and Technology (Korea, Republic of) 
7753 2B Coherent probe-pump-based Brillouin sensor for 100- $\mu \mathrm{m}$ crack detection and 100-km distributed strain and temperature sensing [7753-252]

L. Zou, O. Sezerman, OZ Optics, Ldt. (Canada)

7753 2C Centimeter-range spatial resolution distributed sensing by BOFDA [7753-407]

R. Bernini, Istituto per il Rilevamento Elettromagnetico dell'Ambiente, CNR (Italy);

A. Minardo, L. Zeni, Seconda Univ. degli Studi di Napoli (Italy)

7753 2D Polarization pulling based on stimulated Brillouin scattering in a dual-pump configuration [7753-272]

Z. Shmilovitch, A. Eyal, M. Tur, Tel-Aviv Univ. (Israel); A. Zadok, Bar-Ilan Univ. (Israel);

N. Primerov, S. Chin, L. Thévenaz, Ecole Polytechnique Fédérale de Lausanne (Switzerland)

$77532 \mathrm{E} \quad$ Reduction of measurement time in BOTDA sensors using wavelet shrinkage [7753-369]

M. Amiri Farahani, B. G. Colpitts, E. Castillo-Guerra, Univ. of New Brunswick (Canada)

$77532 \mathrm{~F} \quad$ Self-heterodyne synchronous detection for SNR improvement and distributed Brillouin phase shift measurements in BOTDA sensors [7753-392]

A. Zornoza, D. Olier, A. Loayssa, Univ. Pública de Navarra (Spain)

7753 2G Intensity and phase noise caused by stimulated Brillouin scattering [7753-274]

W. Chen, Z. Meng, National Univ. of Defense Technology (China)

$77532 \mathrm{H} \quad 30 \mathrm{~cm}$ of spatial resolution using pre-excitation pulse BOTDA technique [7753-173]

C. A. Galindez, A. Quintela, M. A. Quintela, J. M. Lopez-Higuera, Univ. de Cantabria (Spain)

$775321 \quad$ Quasi-static strain sensing using molecular spectroscopy [7753-35]

T. T.-Y. Lam, J. H. Chow, D. A. Shaddock, I. C. M. Littler, The Australian National Univ. (Australia); G.-Gagliardi, Istituto Nazionale di Ottica, CNR (Italy); M. B. Gray, National Measurement Institute (Australia); D. E. McClelland, The Australian National Univ. (Australia)

7753 2J Sensing emulsification processes by photon density wave spectroscopy [7753-309]

O. Reich, L. Bressel, R. Hass, Univ. Potsdam (Germany)

$77532 \mathrm{~K} \quad$ Laser induced breakdown spectroscopy algorithm using weights iteration artificial neural network [7753-386]

X. Ma, Z. Zheng, H. Zhao, M. Zhang, Y. Liao, Tsinghua Univ. (China)

$77532 \mathrm{~L} \quad$ Phase-locking of commercial DFB lasers for distributed optical fiber sensing applications [7753-230]

C. D. Rouse, Univ. of New Brunswick (Canada); A. W. Brown, Dark-Pulse Technologies Ltd. (Canada); M. T. V. Wylie, B. G. Colpitts, Univ. of New Brunswick (Canada)

$77532 \mathrm{M} \quad 500 \mathrm{~km}$ remote interrogation of optical sensor arrays [7753-425]

E. Austin, Stingray Geophysical Ltd. (United Kingdom); Q. Zhang, S. Alam, M. Zervas, R. Slavik, P. Petropoulos, Univ. of Southampton (United Kingdom); P. Nash, Stingray Geophysical Ltd. (United Kingdom); D. J. Richardson, Univ. of Southampton (United Kingdom)

775320 Fibre optic distributed differential displacement sensor [7753-10]

M. T. V. Wylie, Univ. of New Brunswick (Canada); A. W. Brown, Dark-Pulse Technologies Ltd.

(Canada); B. G. Colpitts, Univ. of New Brunswick (Canada) 
$77532 \mathrm{P} \quad$ Refractive index sensing based on Mach-Zehnder interferometer formed by three cascaded single-mode fiber tapers [7753-88]

T. Zhu, Chongqing Univ. (China) and Univ. of Ottawa (Canada); D. Wu, M. Deng, D. Duan, Chongqing Univ. (China); Y. Rao, Univ. of Ottawa (China) and Univ. Of Electronic Science and Technology of China (China); X. Bao, Univ. of Ottawa (Canada)

$77532 \mathrm{Q}$ Tunable narrow linewidth and stable frequency laser based on stimulated Rayleigh scattering in non-uniform optical fiber [7753-170]

T. Zhu, X. Bao, L. Chen, Univ. of Ottawa (Canada)

$77532 \mathrm{R} \quad$ Characteristics of stimulated Rayleigh scattering in optical fibers [7753-168]

T. Zhu, X. Bao, L. Chen, H. Liang, Y. Dong, Univ. of Ottawa (Canada)

$77532 \mathrm{~S}$ Millimeter resolution distributed dynamic strain measurements using optical frequency domain reflectometry [7753-335]

A. K. Sang, M. E. Froggatt, S. T. Kreger, D. K. Gifford, Luna Innovations Inc. (United States)

$77532 \mathrm{~T} \quad$ Chaotic lasers for elimination of low-frequency fluctuations of backscattered Rayleigh radiation in distributed fiber optical sensors [7753-300]

V. V. Spirin, C. A. López-Mercado, S. V. Miridonov, Scientific Research and Advanced Studies Ctr. of Ensenada (Mexico); L. Cardoza-Avendaño, R. M. López-Gutiérrez, Univ. Autónoma de Baja California (Mexico); C. Cruz-Hernández, Scientific Research and Advanced Studies Ctr. of Ensenada (Mexico)

$77532 \mathrm{U} \quad$ Distributed temperature monitoring of long distance submarine cables [7753-320] M. Fromme, LIOS Technology GmbH (Germany); W. Christiansen, S. V. Kjaer, DONG Energy (Denmark); W. Hill, LIOS Technology GmbH (Germany)

7753 2V Hybrid TDM/WDM based fiber optic sensor network for perimeter intrusion detection [7753-440]

X. Li, Q. Sun, Z. Sun, J. Wo, M. Zhang, D. Liu, Huazhong Univ. of Science and Technology (China)

$77532 \mathrm{~W} \quad$ Improving the dynamic range in distributed anti-Stokes Raman thermometry by means of susceptibility asymmetry [7753-332]

L. A. Ribeiro, Instituto Nacional de Pesquisas Espaciais (Brazil); J. B. Rosolem, CpqD

Foundation (Brazil); A. O. Toledo, Instituto de Estudos Avançados (Brazil)

$77532 X \quad$ The three point method for measurement of P-OTDR sensor [7753-447]

C. Wu, C. Shang, Z. Li, Beijing Jiaotong Univ. (China)

$77532 \mathrm{Y} \quad$ Measurement of nonlinear refractive index by using input-output characteristics in OFRR nonlinear dynamics [7753-186]

Y. Imai, S. Yamauchi, H. Yokota, S. Wei, Ibaraki Univ. (Japan)

$77532 Z$ Quasi-distributed vibration sensor based on polarization-sensitive measurement [7753-305] N. Linze, P. Tihon, O. Verlinden, P. Mégret, M. Wuilpart, Univ. de Mons (Belgium)

775330 A wide-area fiber sensor network with optical power supply [7753-348] Y. Tanaka, M. Kinoshita, A. Takahashi, T. Kurokawa, Tokyo Univ. of Agriculture and Technology (Japan) 
$775331 \quad$ Fiber optic intrusion detection sensor for physical security system [7753-18]

T. Kumagai, S. Sato, W. Ohnuki, T. Nakamura, Hitachi Cable, Ltd. (Japan)

775332 A new multi-point sensing system based on optical pass switching and remote optical power supply [7753-120]

O. Ogawa, Central Research Institute of Electric Power Industry (Japan)

775333 High performance wavelength demodulator for DFB fiber laser sensor using novel PGC algorithm and reference compensation method [7753-292]

J. He, F. Li, W. Zhang, L. Wang, T. Xu, Y. Liu, Institute of Semiconductors (China)

775334 Low frequency acoustic response of a planar fiber laser cantilever in a fluid [7753-176] G. A. Cranch, G. A. Miller, C. K. Kirkendall, U.S. Naval Research Lab. (United States)

775335 Acoustic sensor based on depressed cladding erbium doped fiber ring laser [7753-25] J. B. Rosolem, CpqD Foundation (Brazil); M. B. Elias, Univ. Estadual de Campinas (Brazil); E. W. Bezerra, CpqD Foundation (Brazil); C. K. Suzuki, Univ. Estadual de Campinas (Brazil)

775336 Ultrasound detection using a tunable low beat-frequency $\mathrm{Er}^{3+}$-doped DBR fiber laser [7753-157]

T. Guo, Jinan Univ. (China) and The Hong Kong Polytechnic Univ. (China); A. C. Wong,

W. Liu, The Hong Kong Polytechnic Univ. (Hong Kong, China); B. Guan, Jinan Univ. (China);

C. Lu, H.-Y. Tam, The Hong Kong Polytechnic Univ. (Hong Kong, China)

775337 Ultra thin fiber laser vector hydrophone [7753-60]

R. Ma, W. Zhang, J. He, F. Li, Y. Liu, Institute of Semiconductors (China)

775338 Full characterization and comparison of phase properties of narrow linewidth lasers operating in the C-band [7753-132]

R. Slavik, Y. Liao, Univ. of Southampton (United Kingdom); E. Austin, Stingray Geophysical Ltd. (United Kingdom); P. Petropoulos, D. J. Richardson, Univ. of Southampton (United Kingdom)

775339 Mode-locked multi-wavelength fiber ring laser using low frequency phase modulation [7753-364]

C. S. Jun, J. K. Ko, S. H. Yoo, B. Y. Kim, Korea Advanced Institute of Science and Technology (Korea, Republic of)

7753 3A Fast method for engineering erbium-doped fiber lasers [7753-375]

J. D. Causado-Bulevas, N. D. Gómez-Cardona, P. Torres, Univ. Nacional de Colombia (Colombia)

7753 3B $\quad 50 \mathrm{~km}$ long distance DFB fiber laser hydrophone system [7753-57]

Z. Chen, J. Ng, A*STAR Institute for Infocomm Research (Singapore); V. Pallayil, National Univ. of Singapore (Singapore)

7753 3C L-band multiwavelength erbium-doped fiber ring laser for sensing applications [7753-242]

R. A. Perez-Herrera, Univ. Pública de Navarra (Spain); A. Ullán, Univ. de Cantabria (Spain);

D. Leandro, M. Fernández-Vallejo, Univ. Pública de Navarra (Spain); M. A. Quintela, Univ. de Cantabria (Spain); A. Loayssa, Univ. Pública de Navarra (Spain); J. M. López-Higuera, Univ. de Cantabria (Spain); M. Lopez-Amo, Univ. Pública de Navarra (Spain) 
7753 3D Fiber laser vector hydrophone: theory and experiment [7753-401]

W. Zhang, F. Zhang, R. Ma, J. He, F. Li, Y. Liu, Institute of Semiconductors (China)

7753 3E Weak injection locked DFB semiconductor laser for optical fiber sensing [7753-398]

H. Zhou, Q. Yao, M. Chen, Z. Meng, National Univ. of Defense Technology (China)

\section{RAYLEIGH TECHNIQUES AND FREQUENCY DOMAIN SENSORS}

$77533 \mathrm{~F}$ Long-range coherent optical frequency domain reflectometry and its applications (Invited Paper) [7753-71]

F. Ito, X. Fan, Y. Koshikiya, NTT Access Network Service Systems Labs. (Japan)

7753 3G Frequency domain simultaneous tone interrogation for faster, sweep-free Brillouin distributed sensing [7753-254]

A. Voskoboinik, J. Wang, A. E. Willner, The Univ. of Southern California (United States); M. Tur, Tel Aviv Univ. (Israel)

$77533 \mathrm{H} \quad$ High-performance Brillouin strain and temperature sensor based on frequency division multiplexing using nonuniform fibers over $75-\mathbf{k m}$ fiber [7753-264]

Y. Dong, X. Bao, L. Chen, Univ. of Ottawa (Canada)

775331 High precision, high sensitivity distributed displacement and temperature measurements using OFDR-based phase tracking [7753-243]

D. K. Gifford, M. E. Froggatt, S. T. Kreger, Luna Innovations Inc. (United States)

7753 3J Ultra high dynamic range coherent optical time domain reflectometry employing frequency division multiplexing [7753-282]

H. lida, Y. Koshikiya, F. Ito, K. Tanaka, NTT Access Network Service Systems Labs. (Japan)

$77533 \mathrm{~K}$ Vibration monitoring with high frequency response based on coherent phase-sensitive OTDR method [7753-236]

Y. Lu, T. Zhu, X. Bao, L. Chen, Univ. of Ottawa (Canada)

NEW FIBERS

7753 3L Fibre lasers for photo-acoustic gas spectroscopy [7753-131]

N. Arsad, Univ. of Strathclyde (United Kingdom) and Univ. Kebangsaan Malaysia (Malaysia);

G. Stewart, Univ. of Strathclyde (United Kingdom)

7753 3M Improved time response for polymer fibre Bragg grating based humidity sensors [7753-331] W. Zhang, D. J. Webb, Aston Univ. (United Kingdom); G.-D. Peng, The Univ. of New South Wales (Australia)

$77533 \mathrm{~N}$ Two-photon excited fluorescence in praseodymium doped fibre and its application in distributed optical fibre sensing of temperature [7753-104]

C. J. Dalzell, T. P. J. Han, I. S. Ruddock, Univ. of Strathclyde (United Kingdom) 
POSTER SESSION: MICROSTRUCTURAL FIBER, NOVEL CONCEPTS, ETC.

775330 Very high polarimetric sensitivity to strain of second order mode of highly birefringent microstructured fibre [7753-465]

T. Nasilowski, Military Univ. of Technology (Poland); K. Skorupski, M. Makara, Maria Curie-Skłodowska Univ. (Poland); G. Statkiewicz-Barabach, Wroclaw Univ. of Technology (Poland); P. Mergo, Maria Curie-Skłodowska Univ. (Poland); P. Marc, L. Jaroszewicz, Military Univ. of Technology (Poland)

$77533 \mathrm{P} \quad$ Low cost pressure sensor system based on polarization-maintaining photonic crystal fiber operating at $850 \mathrm{~nm}$ with CCD interrogator [7753-175]

L. Cho, H. Y. Fu, The Hong Kong Polytechnic Univ. (Hong Kong, China); C. Wu, The Hong Kong Polytechnic Univ. (Hong Kong, China) and Dalian Univ. of Technology (China); C. Lu, H. Y. Tam, The Hong Kong Polytechnic Univ. (Hong Kong, China)

$77533 \mathrm{Q}$ Single-mode 7-cell core hollow core photonic crystal fiber with increased bandwidth [7753-251]

J. K. Lyngsø, C. Jakobsen, H. R. Simonsen, J. Broeng, NKT Photonics A/S (Denmark)

$77533 R \quad$ Side-hole polarization-maintaining photonic crystal fiber for hydrostatic pressure sensing [7753-23]

C. Wu, Dalian Univ. of Technology (China) and The Hong Kong Polytechnic Univ. (Hong Kong, China); J. Li, X. Feng, B.-O. Guan, Jinan Univ. (China); H.-Y. Tam, The Hong Kong Polytechnic Univ. (Hong Kong, China)

775335 Phase sensitivity to axial strain of microstrustured optical silica fibers [7753-68]

Y. Léguillon, Thales Underwater Systems (France), Univ. Europénne de Bretagne (France), and Fonctions Optiques pour les Technologies de l'information, CNRS (France); P. Besnard, Univ. Europénne de Bretagne (France) and Fonctions Optiques pour les Technologies de I'information, CNRS (France); L. Provino, A. Monteville, D. Méchin, D. Trégoat, Plate-forme d'Étude et de Recherche sur les Fibres Optiques Spéciales (France); M. Doisy, F.-X. Launay, Thales Underwater Systems (France)

7753 3т A photonic crystal fiber temperature sensor based on forward stimulated fluorescence emission [7753-130]

X. Li, X. Hong, Shenzhen Univ. (China) and Shenzhen Key Lab. of Sensor Technology (China); Y. Deng, Shenzhen Key Lab. of Sensor Technology (China); Y. Yu, Y. Geng, Shenzhen Univ. (China) and Shenzhen Key Lab. of Sensor Technology (China); H. Wei, W. Tong, Yangtze Optical Fibre and Cable Co., Ltd. (China)

$77533 \mathrm{U}$ High-sensitive temperature sensor based on alcohol-filled highly birefringent photonic crystal fiber loop mirror [7753-136]

S. He, C.-L. Zhao, X. Dong, S. Zhang, S. Jin, China Jiliang Univ. (China); J. Guo, H. Wei, Yangtze Optical Fibre and Cable Co., Ltd. (China)

7753 3V Transversal-force sensor based on supercontinuum generation in photonic crystal fibers [7753-172]

L. H. Chen, R. M. Li, C. C. Chan, L. M. Zhao, Nanyang Technological Univ. (Singapore);

Y. X. Jin, X. Y. Dong, China Jiliang Univ. (China); K. C. Leong, Globalfoundaries Singapore Pte Ltd. (Singapore) 
7753 3W Potential glucose monitoring of blood plasma using hollow core photonic crystal fibre [7753-177]

L. E. Horan, G. Khara, M. Rutowska, A. D. Ellis, F. C. Garcia Gunning, Univ. College Cork (Ireland)

$77533 \mathrm{X} \quad$ Fabrication of polarization-maintaining photonic crystal fiber couplers using $\mathrm{CO}_{2}$ laser irradiation technique [7753-217]

H. Yokota, Y. Ito, H. Kawashiri, Y. Imai, Y. Sasaki, Ibaraki Univ. (Japan)

7753 3Y A hollow-core photonic bandgap fiber polarization controller [7753-220] M. Pang, W. Jin, The Hong Kong Polytechnic Univ. (Hong Kong, China); Y. Yang, BeiHang Univ. (China)

$77533 Z$ Sensing characteristics of birefringent microstructured polymer optical fiber [7753-295] M. K. Szczurowski, Wroclaw Univ. of Technology (Poland); O. Frazão, J. M. Baptista, INESC Porto (Portugal); K. Nielsen, O. Bang, Technical Univ. of Denmark (Denmark);

W. Urbańczyk, Wroclaw Univ. of Technology (Poland)

775340 Thermo-optic effect of an index guiding photonic crystal fiber with elastomer inclusions [7753-367]

C. Markos, National Hellenic Research Foundation (Greece) and Univ. of Patras (Greece); K. Vlachos, Univ. of Patras (Greece); G. Kakarantzas, National Hellenic Research Foundation (Greece)

$775341 \quad$ Liquid crystal orientation control in photonic liquid crystal fibers [7753-394] M. S. Chychłowski, E. Nowinowski-Kruszelnicki, T. R. Woliński, Warsaw Univ. of Technology (Poland) and Military Univ. of Technology (Poland)

775342 Index guiding photonic liquid crystal fibers for application in fiber optic sensing setups [7753-429]

S. Ertman, M. M. Tefelska, M. S. Chychłowski, Warsaw Univ. of Technology (Poland); D. Pysz, Institute of Electronics Materials Technology (Poland); R. Buczyński, Univ. of Warsaw (Poland); E. Nowinowski-Kruszelnicki, R. Dąbrowski, Military Univ. of Technology (Poland); T. R. Woliński, Warsaw Univ. of Technology (Poland)

775343 Photonic crystal fiber integrated microfluidic chip for highly sensitive real-time chemical sensing [7753-38]

D. Z. Y. Yong, A*STAR Singapore Institute of Manufacturing Technology (Singapore) and Nanyang Technological Univ. (Singapore); X. YU, A*STAR Singapore Institute of Manufacturing Technology (Singapore); C. C. Chan, Nanyang Technological Univ. (Singapore); Y. Zhang, A*STAR Singapore Institute of Manufacturing Technology (Singapore); P. Shum, Nanyang Technological Univ. (Singapore)

775344 Photonic crystal fiber couplers based on large mode area fibers [7753-179] P. Marć, K. Stasiewicz, L. R. Jaroszewicz, T. Nasiłowski, M. Szymański, Military Univ. of Technology (Poland)

$775345 \quad$ Enhancing the sensitivity of liquid refractive index sensor based on slow light photonic crystal waveguide [7753-307]

Y. Zhao, H. Huang, Q. Wang, Northeastern Univ. (China) 
775346 Hybrid photonic crystal fiber sensing of high hydrostatic pressure [7753-343]

M. A. R. Franco, Instituto Tecnológico de Aeronáutica (Brazil) and Instituto de Estudos Avançados (Brazil); V. A. Serrão, Instituto de Estudos Avançados (Brazill); T. R. Pitarello, Instituto Tecnológico de Aeronáutica (Brazil); A. Cerqueira S., Jr., Univ. Estadual de Campinas (Brazil)

775347 Intensity-modulated temperature sensor based on the photonic crystal fibers filled with magnetic fluid [7753-270]

Y. Miao, Y. Liu, Tianjin Univ. of Technology (China); B. Liu, Nankai Univ. (China); K. Zhang, Tianjin Univ. of Technology (China); H. Zhang, Q. Zhao, Nankai Univ. (China)

775348 Colloidal photonic crystals self-assembled onto the optical fiber substrates [7753-51] M. Wang, Nanjing Normal Univ. (China); W. Guo, Nanjing Normal Univ. (China) and Changshu Institute of Technology (China); H. Yan, L. Dai, Nanjing Normal Univ. (China)

775349 Bending effect on fiber optic evanescent absorption sensor for sensitivity enhancement in hetero-core structured fiber optic [7753-127]

Y. Takamoto, A. Seki, K. Takagi, H. Sasaki, M. Nishiyama, K. Watanabe, Soka Univ. (Japan)

7753 4A Multimodal interference based on large-core air-clad photonic crystal fibres for simultaneous measurement of multiparameters [7753-365]

S. Silva, J. L. Santos, INESC Porto (Portugal) and Univ. do Porto (Portugal); F. X. Malcata, Instituto Superior da Maia (Portugal) and Ctr. Interdisciplinar Investigação Marinha e Ambiental (Portugal); J. Kobelke, K. Schuster, IPHT Jena (Germany); O. Frazão, INESC Porto (Portugal)

$77534 \mathrm{~B}$ Wearable motion capturing with the flexing and turning based on a hetero-core fiber optic stretching sensor [7753-195]

Y. Koyama, M. Nishiyama, K. Watanabe, Soka Univ. (Japan)

7753 4D A hetero-core fiber optic smart mat sensor for discrimination between a moving human and object on temporal loss peaks [7753-192]

A. Hosoki, M. Nishiyama, Y. Choi, K. Watanabe, Soka Univ. (Japan)

7753 4E Detection of soil gravity water with hetero-core optical fiber sensor [7753-275]

N. Kumekawa, K. Watanabe, Soka Univ. (Japan)

$77534 \mathrm{~F} \quad$ Effect of doping swelling polymer cladding with phthalocyanine dye in plastic optical fiber humidity sensors [7753-40]

M. Morisawa, H. Yokomori, Univ. of Yamanashi (Japan)

7753 4G Low-cost, non-contact displacement sensor based on plastic fiber bundle [7753-167]

D. Tosi, A. Neri, G. Perrone, A. Vallan, Politecnico di Torino (Italy)

$77534 \mathrm{H} \quad$ Self-repairing polymer optical fiber sensor [7753-249]

Y. Song, K. Peters, North Carolina State Univ. (United States)

$775341 \quad$ Twisted optical microfiber for refractive index sensing [7753-293]

C. Liao, D. Wang, X. He, M. Yang, The Hong Kong Polytechnic Univ. (Hong Kong, China) 
$77534 \mathrm{~J} \quad$ Bend sensors based on periodically tapered soft glass fibers [7753-181]

Y. Wang, Univ. of Southampton (United Kingdom) and Harbin Engineering Univ. (China);

D. Richardson, G. Brambilla, X. Feng, M. Petrovich, M. Ding, Z. Song, Univ. of Southampton (United Kingdom)

$77534 \mathrm{~K}$ Effects of aluminum doping concentrations on radiation sensitivity of erbium-doped fibers [7753-395]

T.-S. Peng, Y.-C. Hsieh, L. A. Wang, National Taiwan Univ. (Taiwan); W.-C. Chiou, R.-Y. Liu,

National Space Organization (Taiwan)

$77534 \mathrm{~L} \quad$ Endoscopic optical coherence elastography using acoustic radiation force and bending vibration of optical Fiber [7753-54]

R. Isago, K. Nakamura, Tokyo Institute of Technology (Japan)

$77534 \mathrm{M}$ Fiber optic confocal microscopy using a miniaturized needle-compatible imaging probe [7753-470]

R. S. Pillai, D. Lorenser, D. D. Sampson, The Univ. of Western Australia (Australia)

$77534 \mathrm{~N} \quad$ Temperature monitoring of superconducting wire for quench detection [7753-197]

K. Omichi, Y. Terada, A. Sakamoto, R. Yamauchi, Fujikura Ltd. (Japan)

775340 Ferrule-top cantilever optical fiber sensor for velocity measurements of low speed air flows [7753-212]

A. Cipullo, Second Univ. of Naples (Italy); G. Gruca, K. Heeck, Vrije Univ. (Netherlands);

F. De Filippis, Italian Aerospace Research Ctr. (Italy); D. Iannuzzi, Vrije Univ. (Netherlands);

L. Zeni, Second Univ. of Naples (Italy)

$77534 \mathrm{P} \quad$ In situ monitoring of carbon dioxide emissions from a diesel engine using a mid-infrared optical fibre sensor [7753-262]

E. Lewis, J. Clifford, C. Fitzpatrick, G. Dooly, Univ. of Limerick (Ireland); W. Zhao, T. Sun, K. Grattan, The City Univ. (United Kingdom); J. Lucas, Univ. of Liverpool (United Kingdom); M. Degner, H. Ewald, Univ. Rostock (Germany); S. Lochmann, G. Bramann, Hochschule Wismar (Germany); E. Merlone-Borla, F. Gili, Ctr. Ricerche Fiat S.C.p.A. (Italy)

$77534 \mathrm{Q} \quad$ Low concentration fluorescence sensing in suspended-core fibers [7753-427] E. P. Schartner, H. Ebendorff-Heidepriem, T. M. Monro, The Univ. of Adelaide (Australia)

7753 4R Modeling of polarization mode coupling involved in a capillary optical fibre sensor [7753-180]

C. Paré, S. Caron, P. Paradis, A. Proulx, INO (Canada)

7753 4S Magnetic field sensing using D-fiber coupled Bi:RIG slab [7753-246]

B. Shreeve, R. Selfridge, S. Schultz, Brigham Young Univ. (United States); C. Gaeta, R. Forber, IPITEK, Inc. (United States)

$77534 \mathrm{~T} \quad$ Biosensor application of resonance coupling to thin film planar waveguides on side-polished optical fiber [7753-312]

W. Ecke, Institut für Photonische Technologien e.V. (Germany); A. Andreev, Institute of Solid State Physics (Bulgaria); A. Csaki, K. Kirsch, K. Schroeder, T. Wieduwilt, R. Willsch, Institut für Photonische Technologien e.V. (Germany) 
$77534 \mathrm{U}$ Distance displacement measurement with two-photon absorption process in Si-APD and high-speed optical millimeter wave scanner [7753-349]

Y. Tanaka, D. Meguro, N. Endo, T. Kurokawa, Tokyo Univ. of Agriculture and Technology (Japan)

$77534 \mathrm{~V} \quad$ Stress monitoring in a maxilla model and dentition [7753-418]

M. S. Milczewski, Brazilian Dentistry Association, Parana (Brazil); H. J. Kalinowski,

J. C. C. da Silva, Federal Univ. of Technology (Brazil); I. Abe, Institute of Nanostructures, Nanomodelling and Nanofabrication (Portugal); A. Simões, Univ. de Aveiro (Portugal); A. Saga, Brazilian Dentistry Association, Parana (Brazil)

\section{Part 2}

$77534 \mathrm{~W}$ Development of an integrated fibre optic sensing network for a composite rudder [7753-189]

C. Davis, I. Grabovac, Defence Science and Technology Organisation (Australia); R. Crane, Naval Surface Warfare Ctr. (United States); C. Ratcliffe, U.S. Naval Academy (United States)

$77534 \mathrm{X} \quad$ Fibre optic sensors for high speed hypervelocity impact studies and low velocity drop tests [7753-231]

D. A. Jackson, M. J. Cole, M. J. Burchell, Univ. of Kent (United Kingdom); D. J. Webb, Aston Univ. (United Kingdom)

7753 4Y Evanescent-wave fiber optic sensor: on power transfer from core-cladding interface to fiber end-face [7753-245]

Y. Chiniforooshan, J. Ma, W. J. Bock, Univ. du Québec en Outaouais (Canada)

$77534 Z$ Influence of the lamination process on the strain sensitivity of the fiber sensors embedded in composite materials [7753-410]

P. Lesiak, Warsaw Univ. of Technology (Poland); G. Rajan, Y. Semenova, G. Farrell, Dublin Institute of Technology (Ireland); A. Boczkowska, D. Budaszewski, M. Szelag, A. Domański, T. Woliński, Warsaw Univ. of Technology (Poland)

$775350 \quad$ Numerical modelling of imaging fibre bundles and their application in optical coherence tomography [7753-454]

A. Saglam, H. D. Ford, R. P. Tatam, Cranfield Univ. (United Kingdom)

$775351 \quad$ A dynamic fiber optic strain and power change sensor [7753-32]

S. Liehr, K. Krebber, BAM Federal Institute for Materials Research and Testing (Germany)

775352 Highly sensitive fiber optic inclinometer: easy to transport and easy to install [7753-43] P. Lenke, M. Wendt, K. Krebber, BAM Federal Institute for Materials Research and Testing (Germany); R. Glöłzl, Glötzl Gesellschaft für Baumesstechnik mbH (Germany)

775353 Linear-core-array optical fiber based laser beam shape convertor [7753-48]

L. Yuan, X. Zhu, A. Zhou, Q. Dai, F. Tian, Harbin Engineering Univ. (China) 
775354 Launching the excitation light to a taper externally: comprehensive performance improvement of fiber optic evanescent-wave sensor [7753-52]

H. Chen, China Jiliang Univ. (China); J. Ma, W. J. Bock, Univ. du Québec en Outaouais (Canada)

775355 An improved radiometric wavelength measurement system incorporating fibre comb filters fabricated by $\mathrm{CO}_{2}$ laser irradiation [7753-171]

P. Wang, Univ. of Southampton (United Kingdom) and Dublin Institute of Technology

(Ireland); G. Brambilla, M. Ding, Y. Wang, Univ. of Southampton (United Kingdom);

Y. Semenova, Q. WU, L. Bo, G. Farrell, Dublin Institute of Technology (Ireland)

775356 Turbidity sensor for determination of concentration, ash presence and particle diameter of sediment suspensions [7753-232]

L. Bilro, S. Prats, J. L. Pinto, J. J. Keizer, Univ. de Aveiro (Portugal); R. N. Nogueira, Instituto de Telecomunicações (Portugal)

775357 Fiber ringdown breathing rate sensor [7753-257]

Z. Chen, H. Yim, J. T. Teo, S. H. Ng, A*STAR Institute for Infocomm Research (Singapore)

775358 Optical fiber ferrule-top sensor for humidity measurements [7753-324]

G. Gruca, J. Rector, K. Heeck, D. Iannuzzi, Vrije Univ. Amsterdam (Netherlands)

775359 Micro fluidic channel actuator using optical force induced by evanescent field material coupling [7753-390]

H. Choi, W. Ha, M. Park, K. Oh, Yonsei Univ. (Korea, Republic of)

7753 5A Hydrazine concentration fiber optic reversible sensor [7753-421]

A. Andrawis, Y. Peng, X. Yan, B. Ranjitkar, South Dakota State Univ. (United States)

7753 5B Composite cavity fiber laser sensor based on feedback modulation [7753-387]

J. Zhang, Y. Ge, Q. Cai, Q. Hao, Q. Li, W. Sun, L. Yuan, Harbin Engineering Univ. (China); P. Lu, Communications Research Ctr. Canada (Canada); G. D. Peng, The Univ. of New South Wales (Australia)

7753 5C Highly sensitive refractive index sensor based on cladding mode interference in microtapered SMF [7753-441]

S. M. Tripathi, A. Kumar, Indian Institute of Technology (India); E. Marin, J.-P. Meunier, Lab. Hubert Curien, CNRS, Univ. Lyon and Univ. Jean Monnet Saint-Etienne (France)

$77535 \mathrm{D}$ Structural bending sensor with temperature insensitivity based on a single polarization fiber [7753-27]

M.-S. Yoon, O.-J. Kwon, H.-J. Kim, S. Chu, Y.-G. Han, Hanyang Univ. (Korea, Republic of)

$77535 \mathrm{E}$ High-speed focusing of a liquid microlens using acoustic radiation force [7753-58]

D. Koyama, R. Isago, K. Nakamura, Tokyo Institute of Technology (Japan)

$77535 \mathrm{~F} \quad$ Refractive index measurement by using multimode interference [7753-439]

Y. X. Jin, China Jiliang Univ. (China); C. C. Chan, Nanyang Technological Univ. (Singapore);

Y. Zhao, X. Y. Dong, China Jiliang Univ. (China) 
$77535 \mathrm{G}$ The use of a bent singlemode-multimode-singlemode (SMS) fiber structure for vibration sensing [7753-174]

Q. Wu, Y. Semenova, P. Wang, G. Farrell, Dublin Institute of Technology (Ireland)

$77535 \mathrm{H} \quad$ Investigation of single mode polarization-maintaining fibres for directional transverse force measurement [7753-185]

M. Karimi, City Univ. London (United Kingdom) and Azad Univ. (United Kingdom); F. Surre,

T. Sun, K. T. V. Grattan, City Univ. London (United Kingdom); P. Fonjallaz, Acreo AB (Sweden)

$775351 \quad$ Measurement of sucrose and ethanol concentrations in process streams and effluents of sugarcane bioethanol industry by optical fiber sensor [7753-219]

E. Fujiwara, E. Ono, T. P. Manfrim, J. S. Santos, C. K. Suzuki, Univ. Estadual de Campinas (Brazil)

7753 5J Ultra fast all-optical fiber pressure sensor for blast event evaluation [7753-225]

N. Wu, W. Wang, Y. Tian, C. Niezrecki, X. Wang, Univ. of Massachusetts Lowell (United States)

7753 5K Fibre optic pressure sensor system for high temperature exhaust gas flows [7753-247]

K. Bremer, E. Lewis, G. Leen, B. Moss, J. Leen, Univ. of Limerick (Ireland); S. Lochmann,

I. Mueller, Hochschule Wismar (Germany)

$77535 \mathrm{~L}$ Design of a polarization-insensitive optical fiber probe based on effective parameters [7753-258]

T.-T.-H. Pham, Y.-L. Lo, National Cheng Kung Univ. (Taiwan)

$77535 \mathrm{M}$ Enabling low-cost, high-performance vapor-phase TNT detection by optimizing multimode fiber sensing platform [7753-283]

J. Ma, A. Kos, W. J. Bock, Univ. du Québec en Outaouais (Canada); X. Li, H. Nguyen,

Z. Y. Wang, Carleton Univ. (Canada)

$77535 \mathrm{~N} \quad$ Remote ice detection system for on-board applications based on fiber optics [7753-323]

M. Ruiz-Llata, P. Acedo, Univ. Carlos III de Madrid (Spain)

775350 Fiber optic rotational seismic system for investigation of the rotational events [7753-403] L. R. Jaroszewicz, Z. Krajewski, Military Univ. of Technology (Poland); J. Kowalski, m-Soft Ltd. (Poland); P. Zinówko, Elproma Electronics Ltd. (Poland)

7753 5P Three-component all polarization-maintaining optical fiber vector hydrophone [7753-436] J. Wang, H. Luo, Z. Meng, Y. Hu, National Univ. of Defense Technology (China)

$77535 \mathrm{Q} \quad$ Improving optical fiber current sensor accuracy using artificial neural networks to compensate temperature and minor non-ideal effects [7753-448]

A. C. Zimmermann, M. Besen, L. S. Encinas, Univ. Federal de Santa Catarina (Brazil)

R. Nicolodi, Centrais Elétricas de Santa Catarina S.A. (Brazil)

$77535 R \quad$ Long-gauge strain sensors for underwater and deep-water applications [7753-468]

D. Inaudi, Smartec S.A. (Switzerland)

$77535 S \quad$ Characterization of tapered polymer optical fibers under side illumination for fluorescence sensing applications [7753-59]

C. Pulido, Ó. Esteban, Univ. de Alcalá de Henares (Spain) 
$77535 \mathrm{~T}$ Optical characterisation of RF sputter coated palladium thin films for hydrogen sensing [7753-405]

R. M. Carter, Heriot-Watt Univ. (United Kingdom); P. Morrall, AWE plc (United Kingdom); R. R. J. Maier, Heriot-Watt Univ. (United Kingdom); B. J. S. Jones, S. McCulloch, AWE plc (United Kingdom); J. S. Barton, Heriot-Watt Univ. (United Kingdom)

$77535 \mathrm{U}$ Design and optimization of an optical refractometer for remote measurements via fiber optic cables [7753-430]

S. Khotiaintsev, C. E. García-Guerra, J. E. Morales-Farah, S. Perez-Garcia, L. E. Yam-Ontiveros, Univ. Nacional Autónoma de México (Mexico)

$77535 \mathrm{~V}$ Stripping and splicing polyimide-coated fibers [7753-129]

D. Duke, AFL Telecommunications LLC (United States); Y. Kanda, K. Tobita, R. Yamauchi, Fujikura Ltd. (Japan)

7753 5W Mode field analysis of eccentric optical fibers by conformal mapping [7753-356]

C. Guan, L. Yuan, F. Tian, Q. Dai, X. Tian, Harbin Engineering Univ. (China)

$77535 \mathrm{X}$ Twin-half hollow elliptical core polarization-maintaining fiber for polarization state selective sensing [7753-393]

C. Liu, L. Wang, Z. Liu, Q. Dai, F. Tian, L. Yuan, Harbin Engineering Univ. (China)

$77535 Y \quad$ A compact fiber optic accelerator [7753-42]

F. Peng, X. Li, B. Wu, Y. Yuan, J. Yang, L. Yuan, Harbin Engineering Univ. (China)

$77535 Z$ In-fiber integrated accelerator [7753-284]

F. Peng, X. Li, B. Wu, Y. Yuan, J. Yang, L. Yuan, Harbin Engineering Univ. (China)

775360 Optical path correlator for low-coherence multiplexing fiber optic sensor [7753-49]

Y. Yuan, B. WU, J. Yang, L. Yuan, Harbin Engineering Univ. (China)

775361 Highly sensitive optical fiber oxygen sensor based on dye entrapped core-shell silica nanoparticles [7753-239]

C.-S. Chu, Ming Chi Univ. of Technology (Taiwan); Y.-L. Lo, T.-W. Sung, National Cheng Kung Univ. (Taiwan)

775362 Characteristics of hydrogen gas sensor based on a wavelength division multiplexing fiber coupler [7753-260]

K. S. Park, J. B. Eom, M.-S. Park, Y. H. Kim, S. J. Park, J.-H. Jang, B. H. Lee, Gwangju Institute of Science and Technology (Korea, Republic of)

775363 Fiber optic sensors for monitoring a concrete beam high strain bending test [7753-266] M. Bravo, Univ. Pública de Navarra (Spain); J. Sáenz, M. Bravo-Navas, Eurocontratas S.A. (Spain); M. López-Amo, Univ. Pública de Navarra (Spain)

775364 Radiation-resistance technology for broadband fiber optic source [7753-389]

Y. Yang, X. Suo, M. Yang, X. Shi, W. Jin, BeiHang Univ. (China) 
775365 Construction optimization of the sensors used in point fluorescence investigation of cancer-changed tissues [7753-344]

L. Kłonowski, E. Bereś-Pawlik, Wroclaw Univ. of Technology (Poland); M. Rząca, R. Czarnecki, Regional Specialised Hospital (Poland)

$775366 \quad \mathrm{CO}_{2}$ phase study using an optical fiber refractometer [7753-415]

D. Prada, Pontificia Univ. Católica do Rio de Janeiro (Brazil); C. Martelli, Federal Univ. of Technology (Brazil); C. C. Kato, A. M. B. Braga, M. S. P. Gomes, Pontificia Univ. Católica do Rio de Janeiro (Brazil)

775367 Low loss arc splicing of silica microfibers [7753-434]

C.-D. Chang, S.-M. Chuo, L. A. Wang, National Taiwan Univ. (Taiwan)

775368 Performance characterization of an intensity-modulated fiber optic displacement sensor [7753-13]

E. A. Moro, Univ. of California, San Diego (United States) and Los Alamos National Lab. (United States); M. D. Todd, Univ. of California, San Diego (United States); A. D. Puckett, Los Alamos National Lab. (United States)

775369 Fiber optic hydrogen sensor resisting temperature interference [7753-16]

Y. Zhang, Q. S. Li, Z. Zhuang, Institute of Structural Mechanics (China); M. Zhang, Z. Yang,

Y. B. Liao, Tsinghua Univ. (China)

7753 6A Light scattering measurements for quantifying biological cell concentration: an optimization of opto-geometric parameters [7753-70]

A. G. Mignani, L. Ciaccheri, A. A. Mencaglia, Istituto di Fisica Applicata Nello Carrara, CNR (Italy); L. Giannelli, Hospitex Diagnostics srl (Italy)

7753 6B High pressure measurement by nonadiabatic tapered optical fiber sensor for downhole application [7753-209]

M. I. Zibaii, H. Latifi, M. Kheiri, H. Pourbeyram, S. Nouri Joubari, A. Ahmadlou, M. Karami, Shahid Beheshti Univ. (Iran, Islamic Republic of)

7753 6C Nonadiabatic tapered optical fiber sensor for measuring interaction nicotine with DNA [7753-200]

M. I. Zibaii, H. Latifi, H. Pourbeyram, M. Gholami, Z. Taghipour, Z. Saeedian, S. M. Hosseini, Shahid Beheshti Univ. (Iran, Islamic Republic of)

7753 6D Spectral intensities and phase distributions of supercontinuum pulses generated in low-dispersion fibers [7753-294]

H. Sone, Kitami Institute of Technology (Japan); D. Yoshitomi, X. Zhou, National Institute of Advanced Industrial Science and Technology (Japan) and Japan Science and Technology Agency (Japan); K. Kikuchi, R. Kasahara, Ibaraki Univ. (Japan); F. Abrishamian, Osaka Electro-Communication Univ. (Japan); S. Nakamura, Ibaraki Univ. (Japan); Y. Harada, Kitami Institute of Technology (Japan); K. Torizuka, National Institute of Advanced Industrial Science and Technology (Japan) and Japan Science and Technology Agency (Japan)

7753 6E Optical fibers and sensors for biomedical applications: bend effects [7753-304] S. A. Wade, A. C. Thompson, W. G. A. Brown, D. F. Robertson, P. R. Stoddart, Swinburne Univ. of Technology (Australia) 
$77536 \mathrm{~F}$ Measurement of the velocities in the transient acceleration process using all-fiber photonic Doppler velocimetry [7753-460]

J. Wang, C. Wu, Beijing Jiaotong Univ. (China); H. Song, Institute of Mechanics (China);

T. Yu, J. Xu, Beijing Jiaotong Univ. (China)

7753 6G Two-beam fiber laser Doppler velocimeter enabling velocity distribution measurement of liquid flow [7753-306]

A. L. Bin Muhamad, A. Ugajin, O. Mikami, Tokai Univ. (Japan); T. Okazaki, Y. Yoshikuni,

Kitasato Univ. (Japan)

$77536 \mathrm{H} \quad$ Induction heating assisted optical fiber bonding and sealing technique [7753-334]

P. Niewczas, G. Fusiek, Univ. of Strathclyde (United Kingdom)

\section{PCF SENSORS}

775361 DNA probe detection within 3D hydrogel matrix in a hollow core photonic crystal fibre [7753-417]

M. Rutowska, J. Lu, F. C. Garcia-Gunning, A. D. Ellis, Tyndall National Institute (Ireland) and Univ. College Cork (Ireland)

$77536 \mathrm{~J}$ Introduction of birefringence into photonic crystal fibers [7753-221]

J. Ju, W. Jin, The Hong Kong Polytechnic Univ. (Hong Kong, China); Y. Yang, BeiHang Univ. (China)

$77536 \mathrm{~K}$ High performance interrogation of long period fiber grating sensor with wavelength scanning and Fourier analysis [7753-184]

A. Wada, S. Tanaka, N. Takahashi, National Defense Academy (Japan)

$77536 \mathrm{~L} \quad$ Liquid crystal long-period fiber grating as a sensing element for electric field and temperature measurements [7753-472]

A. Czapla, Univ. du Québec en Outaouais (Canada) and Warsaw Univ. of Technology (Poland); W. J. Bock, Univ. du Québec en Outaouais (Canada); T. R. Woliński, Warsaw Univ. of Technology (Poland); R. Dabrowski, E. Nowinowski-Kruszelnicki, Military Univ. of Technology (Poland)

\section{FBG SENSORS}

7753 6M Regenerated draw tower grating (DTG) temperature sensors [7753-37]

E. Lindner, IPHT Jena (Germany) and FBGS Technologies GmbH (Germany); J. Canning, The Univ. of Sydney (Australia); C. Chojetzki, FBGS Technologies GmbH (Germany); S. Brückner, M. Becker, M. Rothhardt, H. Bartelt, IPHT Jena (Germany)

7753 6N Femtosecond laser inscribed Bragg sensor in Terfenol-D coated optical fibre with ablated microslot for the detection of static magnetic fields [7753-237]

G. N. Smith, T. Allsop, Aston Univ. (United Kingdom); K. Kalli, C. Koutsides, Cyprus Univ. of Technology (Cyprus); R. Neal, Univ. of Plymouth (United Kingdom); K. Sugden, Aston Univ. (United Kingdom); P. Culverhouse, Univ. of Plymouth (United Kingdom); I. Bennion, Aston Univ. (United Kingdom) 
775360 Transverse load sensing with a tilted fiber Bragg grating compressed between conforming elastomers [7753-06]

L.-Y. Shao, Carleton Univ. (Canada) and China Jiliang Univ. (China); J. Albert, Carleton Univ. (Canada)

$77536 \mathrm{P}$ Experimental validation of a numerically determined multi-axial strain transfer from CFRP-laminates to embedded Bragg sensors [7753-233]

G. Luyckx, E. Voet, N. Lammens, W. De Waele, J. Degrieck, Univ. Gent (Belgium)

$77536 \mathrm{Q}$ Numerical modeling of complex femtosecond laser inscribed fiber gratings: comparison with experiment [7753-238]

C. Koutsides, Cyprus Univ. of Technology (Cyprus) and Aston Univ. (United Kingdom); K. Kalli, Cyprus Univ. of Technology (Cyprus); D. J. Webb, L. Zhang, Aston Univ. (United Kingdom)

$77536 R \quad$ Regenerated fibre Bragg gratings used to map internal reaction temperatures of a modified chemical vapour deposition (MCVD) optical fibre preform lathe [7753-303]

M. L. Åslund, The Univ. of Sydney (Australia); A. Canagasabey, Y. Liu, The Univ. of New South Wales (Australia); K. Cook, J. Canning, The Univ. of Sydney (Australia); G.-D. Peng, The Univ. of New South Wales (Australia)

\section{MULTIMODE EFFECTS}

775365 The use of fibre optic sensors to compare the internal strains and pressures produced by different Lamb wave modes [7753-450]

G. Thursby, B. Culshaw, Univ. of Strathclyde (United Kingdom)

$77536 \mathrm{~T} \quad$ Utilisation of thermal annealing to record multiplexed FBG sensors in multimode microstructured polymer optical fibre [7753-327]

I. P. Johnson, D. J. Webb, Aston Univ. (United Kingdom); K. Kalli, Cyprus Univ. of Technology (Cyprus)

$77536 \mathrm{U}$ Target delivery to sensors by using generated uniform three fiber pseudo Bessel beams from one source [7753-381]

J. Kim, S. Lee, Y. Jeong, K. Oh, Yonsei Univ. (Korea, Republic of)

7753 6V Development of a FBG vortex flow sensor for high-temperature applications [7753-400] L. K. Cheng, W. Schiferli, R. A. Nieuwland, TNO Science and Industry (Netherlands);

A. Franzen, J. J. den Boer, Shell International Exploration and Production (Netherlands);

T. H. Jansen, TNO Science and Industry (Netherlands)

\section{BIOMEDICAL APPLICATIONS}

$77536 \mathrm{~W}$ 3D visualization of tissue microstructures using optical coherence tomography needle probes [7753-469]

R. W. Kirk, R. A. Mclaughlin, B. C. Quirk, A. Curatolo, D. D. Sampson, The Univ. of Western Australia (Australia)

$77536 \mathrm{X} \quad$ Fiber-based broadband ultrasound detector for photoacoustic imaging [7753-39] H. Grün, T. Berer, K. Felbermayer, P. Burgholzer, RECENDT GmbH (Austria); G. Paltauf, Karl-Franzens Univ. (Austria) 
77536 A A novel optical-fiber based surface plasmon resonance sensing architecture and its application to gastric cancer diagnostics [7753-194]

A. Francois, J. Boehm, M. Penno, P. Hoffmann, T. M. Monro, Univ. of Adelaide (Australia)

$775362 \quad$ Miniature temperature insensitive fiber optic sensors for minimally invasive surgical devices [7753-216]

G. Rajan, D. Callaghan, Y. Semenova, G. Farrell, Dublin Institute of Technology (Ireland)

$775370 \quad$ High sensitivity interferometric polymer optical fiber ultrasound sensors for optoacoustic imaging and biomedical application [7753-446]

D. Gallego, H. Lamela, Univ. Carlos III de Madrid (Spain)

775371 Dynamic analysis for mental sweating of a group of eccrin sweat glands on a human fingertip by optical coherence tomography [7753-79]

M. Ohmi, M. Tanigawa, Y. Wada, M. Haruna, Osaka Univ. (Japan)

POSTER SESSION: BRAGG GRATINGS, LONG PERIOD GRATINGS, SPECIALIZED GRATINGS

775372 Fiber Bragg grating sensor system using single-mode wavelength swept light source [7753-128]

T. Saitoh, K. Nakamura, H. Furukawa, M. Koshihara, Anritsu Corp. (Japan)

$775373 \quad$ Fiber Bragg grating transverse load sensors using suspended core fibers for directional dependent strain measurement [7753-250]

C. M. Jewart, T. Chen, K. P. Chen, Univ. of Pittsburgh (United States); E. Lindner, J. Fiebrandt, M. Rothhardt, K. Schuster, J. Kobelke, H. Bartelt, IPHT Jena (Germany)

775374 Fibre Bragg gratings subject to high strain at high frequencies [7753-164]

D. A. Jackson, Univ. of Kent (United Kingdom)

775375 Displacement monitoring of switch track and its slab on a bridge of high speed railway monitored by FBG [7753-380]

W. Li, H. Li, J. Cheng, X. Huang, J. Pan, C. Zhou, M. Yang, Wuhan Univ. of Technology (China)

775376 Single coherence peak extraction among synthesized periodical peaks by different beat frequencies for elongation of measurement range in multiplexed long-length distributed FBG sensors [7753-193]

K. Kajiwara, Z. He, K. Hotate, The Univ. of Tokyo (Japan)

775377 Fiber Bragg grating interrogator for demonstration of spaceborne applications [7753-333] M. R. Rößner, M. S. Müller, T. C. Buck, A. W. Koch, Technische Univ. München (Germany)

775378 Magnetic field sensor based on magnetic fluid with side-polished fiber Bragg grating [7753-55]

J. Dai, M. Yang, Wuhan Univ. of Technology (China)

775379 Static and dynamic strain fiber Bragg grating sensor interrogation using a monolithically integrated echelle diffractive grating [7753-183]

H. Guo, Univ. of Ottawa (Canada); G. Xiao, National Research Council Canada (Canada);

N. Mrad, National Defence Headquarters (Canada); J. Yao, Univ. of Ottawa (Canada) 
7753 7A Temperature-independent strain sensor based on four-wave mixing using Raman FBG laser sensor with cooperative Rayleigh scattering [7753-263]

H. F. Martins, M. B. Marques, O. Frazão, INESC Porto (Portugal)

7753 7B Remotely tuneable optical filter based on polymer fibre Bragg grating [7753-321]

W. Zhang, D. J. Webb, Aston Univ. (United Kingdom); G.-D. Peng, The Univ. of New South Wales (Australia)

7753 7C Free water in fuel sensor using fiber long period grating [7753-318]

W. Zhang, S. Grice, K. Sugden, I. Bennion, Aston Univ. (United Kingdom)

7753 7D Hydrogen detection in high pressure gas mixtures using a twin hole fibre Bragg grating [7753-342]

D. Grobnic, S. J. Mihailov, R. B. Walker, G. Cuglietta, C. W. Smelser, Communications Research Ctr. Canada (Canada)

$77537 \mathrm{E} \quad$ Probing of sapphire fiber Bragg gratings using intrinsic black-body radiation [7753-268] D. Grobnic, S. J. Mihailov, C. W. Smelser, Communications Research Ctr. Canada (Canada)

$77537 \mathrm{~F} \quad$ Benchmark for standard and computationally intelligent peak detection algorithms for fiber Bragg grating sensors [7753-345]

L. H. Negri, Santa Catarina State Univ. (Brazil); H. J. Kalinowski, Federal Univ. of Technology

(Brazil); A. S. Paterno, Santa Catarina State Univ. (Brazil)

$77537 \mathrm{G}$ Optimal design and implementation of a temperature and strain optical transducer using FBGs and fiber taper hybrid structure [7753-347]

A. Quintela, L. Rodriguez, M. I. Barquin, C. Galindez, M. A. Quintela, J. M. Lopez-Higuera, Univ. de Cantabria (Spain)

$77537 \mathrm{H} \quad$ Application of simultaneous strain and temperature measurement technique using polarization maintaining fiber Bragg grating for distributed sensing based on OFDR [7753-353]

D. Wada, H. Murayama, The Univ. of Tokyo (Japan); H. Igawa, Japan Aerospace Exploration Agency (Japan); K. Omichi, Fujikura Ltd. (Japan); K. Kageyama, The Univ. of Tokyo (Japan)

7753 7I Fiber Bragg grating interrogation technique for remote sensing (100km) using a hybrid Brillouin-Raman fiber laser [7753-08]

M. Fernandez-Vallejo, D. Leandro, A. Loayssa, M. Lopez-Amo, Univ. Pública de Navarra (Spain)

7753 7J Polarization-switching FBG interrogator for wavelength-encoded polarization-sensitive measurements [7753-33]

P. Orr, P. Niewczas, Univ. of Strathclyde (United Kingdom)

7753 7K Microfiber Bragg grating for liquid-level variation sensing [7753-123]

B. Lin, S. C. Tjin, Nanyang Technological Univ. (Singapore); Y. Zhang, Huazhong Univ. of Science and Technology (China); B. Dong, J. Hao, Institute for Infocomm Research (Singapore) 
$77537 \mathrm{~L} \quad$ Highly birefringent photonic bandgap Bragg fiber loop mirror for sensing applications [7753-140]

M. S. Ferreira, J. M. Baptista, INESC Porto (Portugal); P. Roy, R. Jamier, S. Février, Xlim, CNRS, Univ. de Limoges (France); O. Frazão, INESC Porto (Portugal)

$77537 \mathrm{M}$ Fibre Bragg grating vibration transducer based on novel mechanical sensing element for monitoring applications [7753-156]

S. Andresen, F. K. Nielsen, T. R. Licht, M. N. Rasmussen, M. Kirkelund, Brüel \& Kjaer Sound \& Vibration Measurement A/S (Denmark)

$77537 \mathrm{~N}$ Dual-polarization distributed Bragg reflector fiber lasers for hydrostatic pressure measurement [7753-202]

Y. Zhang, C. WU, Y.-N. Tan, Dalian Univ. of Technology (China); B.-O. Guan, Jinan Univ. (China)

775370 RF-frequency-division multiplexing of polarimetric fiber grating laser sensors [7753-26] Y. Zhang, Y.-N. Tan, Dalian Univ. of Technology (China); B.-O. Guan, Jinan Univ. (China)

$77537 P \quad$ Realization of nano-order static strain resolution in FBG sensors using narrow linewidth tunable laser sources: theoretical analysis [7753-227]

Q. Liu, Z. He, T. Tokunaga, K. Hotate, The Univ. of Tokyo (Japan)

$77537 Q \quad$ High-speed full-spectrum fiber Bragg gratings interrogator system and testing [7753-248]

S. Chadderdon, R. Selfridge, S. Schultz, Brigham Young Univ. (United States); S. Webb,

C. Park, K. Peters, M. Zikry, North Carolina State Univ. (United States)

$77537 R \quad$ A novel FBG-based fence with high sensitivity and low nuisance alarm rate [7753-112] H. WU, Y. Rao, S. Li, X. LU, Y. WU, Univ. of Electronic Science and Technology of China (China)

775375 A fast response tilted fiber Bragg grating fluid refractometer using an exposed-hole microstructured optical fiber [7753-125]

G. Wang, J. Liu, Z. Zheng, J. Xiao, J. Zhang, BeiHang Univ. (China)

$77537 \mathrm{~T}$ A fast response suspended core fiber optical gas sensor with side-opening and micro-holes configurations [7753-133]

G. Wang, J. Liu, Z. Zheng, J. Xiao, J. Zhang, BeiHang Univ. (China)

$77537 \mathrm{~V}$ FBG-based vibration measurement of rotating structure using optical fiber rotary joint [7753-126]

N. Takahashi, S. Tanaka, A. Wada, National Defense Academy (Japan)

$77537 \mathrm{~V} \quad$ Linearly chirped and weakly tilted fiber Bragg grating edge filters for in-fiber sensor interrogation [7753-143]

T. Guo, Jinan Univ. (China) and The Hong Kong Polytechnic Univ. (China); H.-Y. Tam, The Hong Kong Polytechnic Univ. (Hong Kong, China); J. Albert, Carleton Univ. (Canada)

$77537 \mathrm{~W}$ Fast wavelength-swept dispersion-tuned fiber laser over $500 \mathrm{kHz}$ using a wideband chirped fiber Bragg grating [7753-196]

S. Yamashita, Y. Takubo, The Univ. of Tokyo (Japan) 
$77537 X \quad$ Etched fiber Bragg grating sensing system thermically assisted for analysis of water- ethanol mixtures [7753-213]

F. K. Coradin, G. R. C. Possetti, R. C. Kamikawachi, M. Muller, J. L. Fabris, Federal Univ. of

Technology (Brazil)

$77537 Y \quad$ Linear FBG interrogation with a wavelength-swept fiber laser and a volume phase grating spectrometer [7753-214]

H. Kim, M. Song, Chonbuk National Univ. (Korea, Republic of)

$77537 Z$ Three parameters simultaneous measurement with a single TFBG [7753-234]

N. J. Alberto, Univ. de Aveiro (Portugal); C. A. Marques, Instituto de Telecomunicações (Portugal); P. F. Antunes, J. L. Pinto, Univ. de Aveiro (Portugal); R. N. Nogueira, Instituto de Telecomunicações (Portugal)

775380 Thermal characterization of FBG strain gauges for the monitoring of the cupola of Duomo di Milano [7753-310]

A. Cigada, L. Comolli, A. Giussani, F. Roncoroni, F. Zenucchi, Politecnico di Milano (Italy)

775381 Performance of a high-temperature sensor based on regenerated fiber Bragg gratings [7753-328]

D. Barrera, Univ. Politécnica de Valencia (Spain); V. Finazzi, J. Villatoro, ICFO-Institut de Ciencies Fotoniques (Spain); S. Sales, Univ. Politécnica de Valencia (Spain); V. Pruneri, ICFO-Institut de Ciencies Fotoniques (Spain) and ICREA-Institució Catalana de Recerca i Estudis Avançats (Spain)

775382 Strain monitoring in power cables of offshore wind energy plants with femtosecond laser inscribed fibre Bragg gratings [7753-408]

J. Burgmeier, Technische Univ. Clausthal (Germany); P. Funken, Draka Industrial Cable GmbH (Germany); W. Schade, Technische Univ. Clausthal (Germany) and Fraunhofer Heinrich Hertz Institute (Germany)

775383 Resonant hydrophones based on coated fiber Bragg gratings. Part II: experimental analysis [7753-411]

M. Moccia, M. Consales, Univ. degli Studi del Sannio (Italy); A. Iadicicco, Univ. degli Studi di Napoli Parthenope (Italy); M. Pisco, Univ. degli Studi del Sannio (Italy); M. Giordano, Istituto per i Materiali Compositi e Biomedici, CNR (Italy); A. Cutolo, A. Cusano, Univ. degli Studi del Sannio (Italy)

775384 Resonant hydrophones based on coated fiber Bragg gratings. Part I: numerical analysis [7753-371]

M. Moccia, M. Pisco, A. Cutolo, V. Galdi, A. Cusano, Univ. degli Studi del Sannio (Italy)

775385 Impact of hydrogen-induced effects on optical fiber Bragg gratings [7753-416] C. Martelli, Federal Univ. of Technology (Brazil); A. Mendez, MCH Engineering LLC (United States); A. L. C. Triques, Petrobras Research Ctr. (Brazil); A. M. B. Braga, Pontificia Univ. Católica do Rio de Janeiro (Brazil); J. Canning, K. Cook, The Univ. of Sydney (Australia); R. Llerena, V. Takahashi, Pontificia Univ. Católica do Rio de Janeiro (Brazil) 
775386 Miniature fiber Bragg grating strain rosette based on lossless tapers [7753-420] D. Viegas, Univ. do Porto (Portugal) and INESC Porto (Portugal); M. C. Navarrete, N. Díaz-Herrera, A. González-Cano, Univ. Complutense de Madrid (Spain); J. L. Santos, Univ. do Porto (Portugal) and INESC Porto (Portugal); F. M. Araújo, INESC Porto (Portugal) and FiberSensing (Portugal)

775387 Monitoring the hysteresis effects in the strain-stress curve of carbon fiber reinforced laminates by FBG technology [7753-424]

H. Zhang, L.C. Pegasus Corp. (United States); M. Ghandehari, A. Sidelev, New York Univ. Polytechnic Institute (United States); R. Bazhanski, Independent Contractor (United States); P. Wang, New York Univ. Polytechnic Institute (United States); J. Xie, J. Zou, L.C. Pegasus Corp. (United States); E. Lui, New York Univ. Polytechnic Institute (United States); D. Li, F. Fang, L.C. Pegasus Corp. (United States); H.-L. Cui, New York Univ. Polytechnic Institute (United States); X. Wang, Univ. of Massachusetts Lowell (United States)

775388 Cognitive fiber Bragg grating sensors system based on fiber Fabry-Pérot tunable filter technology [7753-422]

H. Zhang, L.C. Pegasus Corp. (United States); P. Wang, Polytechnic Institute of NYU (United States); J. Zou, J. Xie, L.C. Pegasus Corp. (United States); H. Cui, Polytechnic Institute of NYU (United States)

775389 Response of some pi-phase-shifted Bragg gratings to elevated pressure [7753-459] H. K. Bal, N. M. Dragomir, F. Sidiroglou, Victoria Univ. (Australia); S. A. Wade, Swinburne Univ. of Technology (Australia); G. W. Baxter, S. F. Collins, Victoria Univ. (Australia)

7753 8B Fiber Bragg grating microphone system for condition-based maintenance of industrial facilities [7753-98]

D. Tosi, M. Olivero, G. Perrone, A. Vallan, Politecnico di Torino (Italy)

$77538 \mathrm{C} \quad$ High speed high-resolution fiber Bragg grating sensing system for monitoring of weigh-in-motion devices [7753-103]

D. Tosi, M. Olivero, G. Perrone, A. Vallan, Politecnico di Torino (Italy)

7753 8D Wheel flat detection in high-speed railway systems using fiber Bragg gratings [7753-428] M. L. Filograno, P. Corredera, Consejo Superior de Investigaciones Científicas (Spain); M. Gonzalez-Herraez, Univ. de Alcalá de Henares (Spain); M. Rodríguez-Plaza,

A. Andrés-Alguacil, Administrador de Infraestructuras Ferroviarias (Spain)

$77538 \mathrm{E} \quad$ All-fibre twist sensor system based on $45^{\circ}$ and $81^{\circ}$ tilted fibre gratings [7753-91] Z. Yan, A. Adebayo, K. Zhou, L. Zhang, D. Webb, Aston Univ. (United Kingdom)

$77538 \mathrm{~F} \quad$ Numerical comparison of peak detection algorithms for the response of FBG in non-homogeneous strain fields [7753-311]

L. Comolli, A. Micieli, Politecnico di Milano (Italy)

$77538 \mathrm{G}$ Nickel plating of FBG strain sensors for nuclear applications [7753-330]

M. Perry, P. Niewczas, Univ. of Strathclyde (United Kingdom); M. Johnston, Univ. of Strathclyde (United Kingdom) and EDF Energy plc (United Kingdom); J. Mackersie, Univ. of Strathclyde (United Kingdom) 
$77538 \mathrm{H} \quad$ Nonlinear-programming optimized fiber Bragg grating based force-torque-sensor with six degrees of freedom [7753-453]

M. S. Müller, L. Hoffmann, T. C. Buck, R. Wojtech, A. W. Koch, Technische Univ. München (Germany)

$775381 \quad$ Pressure sensor using carbon fiber laminate tube and fiber Bragg grating [7753-17]

D. Song, J. Zou, J. Xie, L.C. Pegasus Corp. (United States); Z. Chen, Jilin Univ. (China); H. Cui, L.C. Pegasus Corp. (United States)

$77538 \mathrm{~J} \quad$ Bragg wavelength-insensitive fiber Bragg grating ultrasound detection system based on a fiber ring laser [7753-20]

H. Tsuda, National Institute of Advanced Industrial Science and Technology (Japan)

7753 8K Side polished fiber Bragg grating sensor for simultaneous measurement of refractive index and temperature [7753-261]

Z. Chen, J. Tang, R. Fan, Y. Zhong, J. Zhang, S. Li, Jinan Univ. (China)

$77538 \mathrm{~L} \quad$ Fiber Bragg grating cantilever sensor system for fluid flow monitoring with temperature compensation [7753-406]

P. LU, Q. Chen, Memorial Univ. of Newfoundland (Canada)

7753 8M Temperature-insensitive 2D tilt sensor with two chirped fiber Bragg gratings [7753-108] L. Hu, X. Dong, S. Zhang, S. Jin, China Jiliang Univ. (China); C. C. Chan, Nanyang Technological Univ. (Singapore)

$77538 \mathrm{~N} \quad$ 100-km long distance FBG vibration sensor based on matching filter demodulation [7753-161]

J. Hu, National Univ. of Singapore (Singapore); Z. Chen, J. T. Teo, A*STAR Institute for Infocomm Research (Singapore); C. YU, National Univ. of Singapore (Singapore) and A*STAR Institute for Infocomm Research (Singapore)

775380 Hydrogen sensor based on side-polished fiber Bragg gratings coated with thin palladium film [7753-41]

H. Liu, M. Yang, J. Dai, k. Cao, H. Liao, P. Zhang, Wuhan Univ. of Technology (China)

7753 8P Liquid core fibre Bragg grating based refractive index sensor formed by femtosecond assisted chemical etching technique [7753-211]

P. Saffari, Z. Yan, K. Zhou, L. Zhang, I. Bennion, Aston Univ. (United Kingdom)

$77538 Q \quad$ Monitoring the junction temperature of an IGBT through direct measurement using a fiber Bragg grating [7753-271]

J. P. Bazzo, T. Lukasievicz, M. Vogt, V. de Oliveira, H. J. Kalinowski, J. C. Cardozo da Silva, Federal Univ. of Technology (Brazil)

7753 8R Fiber Bragg grating sensors embedded in concrete samples for a normalized fire test [7753-329]

A. Bueno, B. Torres, D. Barrera, P. Calderón, Univ. Politécnica de Valencia (Spain); J. M. Lloris, M. J. López, Instituto Tecnologico de la Construcción (Spain); S. Sales, Univ. Politécnica de Valencia (Spain) 
7753 8S Simulation of FBG sensing networks using CDM + SDM [7753-362]

D. Li, Q. Jiang, Q. Sui, Shandong Univ. (China)

$77538 \mathrm{~T} \quad$ FBG sensor system based on wavelength-swept active mode-locking laser with RSOA gain medium [7753-198]

H.-J. Kim, H. D. Lee, M. Y. Jeong, C.-S. Kim, Pusan National Univ. (Korea, Republic of);

J. H. Lee, Univ. of Seoul (Korea, Republic of)

$77538 \mathrm{U}$ Cladding modes FBG curvature sensor based on a core misaligned splice [7753-383]

C. JesUs, INESC Porto (Portugal) and Univ. da Madeira (Portugal); P. A. S. Jorge, INESC Porto (Portugal); J. M. Baptista, Univ. da Madeira (Portugal) and INESC Porto (Portugal); O. Frazão, INESC Porto (Portugal)

$77538 \mathrm{~V}$ Simple CW correlation OTDR for interrogation of multiplexed low-reflectivity FBG sensors [7753-431]

M. G. Shlyagin, A. Arias, Ctr. de Investigación Científica y de Educación Superior de Ensenada (Mexico)

$77538 \mathrm{~W}$ Monitoring of vacuum assisted resin transfer moulding (VARTM) process with superimposed fiber-Bragg gratings [7753-99]

S. Triollet, Lab. Hubert Curien, CNRS (France), Univ. de Toulouse (France), and Ecole des Mines d'Albi (France); L. Robert, Univ. de Toulouse (France) and Ecole des Mines d'Albi

(France); E. Marin, Y. Ouerdane, Lab. Hubert Curien, CNRS (France)

7753 8X 870nm Bragg grating in single mode TOPAS microstructured polymer optical fibre [7753-166] W. Yuan, Technical Univ. of Denmark (Denmark); D. J. Webb, Aston Univ. (United Kingdom); K. Kalli, Cyprus Univ. of Technology (Cyprus); K. Nielsen, A. Stefani, H. K. Rasmussen, O. Bang, Technical Univ. of Denmark (Denmark)

$77538 Y$ Acousto-optic control of the LPG spectrum for sensing applications [7753-223]

R. A. Oliveira, G. R. C. Possetti, Federal Univ. of Technology (Brazil); C. A. F. Marques, Instituto de Telecomunicações (Portugal); P. T. Neves, Jr., Federal Univ. of Technology (Brazil); C. A. Bavastri, Federal Univ. of Paraná (Brazil); R. C. Kamikawachi, J. L. Fabris, M. Muller, Federal Univ. of Technology (Brazil); R. N. Nogueira, Instituto de Telecomunicações (Portugal); J. Canning, The Univ. of Sydney (Australia); A. A. P. Pohl, Federal Univ. of Technology (Brazil)

775382 Tapered long-period fiber gratings working in inverted mode through all-fiber ring shaped illumination [7753-372]

D. Paladino, Univ. degli Studi del Sannio (Italy); A. Iadicicco, S. Campopiano, Univ. degli Studi di Napoli Parthenope (Italy); A. Cutolo, Univ. degli Studi del Sannio (Italy); W. J. Bock, Univ. du Québec en Outaouais (Canada); A. Cusano, Univ. degli Studi del Sannio (Italy)

775390 Response of hydrogel coated cascaded long period gratings to relative humidity [7753-21] X. Yu, Heilongjiang Univ. (China) and The Hong Kong Polytechnic Univ. (Hong Kong, China); M. Zhang, Tsinghua Univ. (China); S. Liu, Heilongjiang Univ. (China); Y. Liao, Tsinghua Univ. (China); W. Jin, The Hong Kong Polytechnic Univ. (Hong Kong, China)

775391 Photonic-crystal-fiber-based surface long-period fiber grating for simultaneous measurement of temperature and ambient index [7753-56]

H.-J. Kim, O.-J. Kwon, S. B. Lee, Y.-G. Han, Hanyang Univ. (Korea, Republic of) 
775392 Flow cell with hybrid LPG and FBG optical fibre sensor for refractometric measurements [7753-240]

F. Baldini, M. Brenci, Istituto di Fisica Applicata Nello Carrara, CNR (Italy); F. Chiavaioli, Univ. degli Studi di Siena (Italy); R. Falciai, C. Trono, Istituto di Fisica Applicata Nello Carrara, CNR (Italy)

775393 Femtosecond laser inscribed superstructure fibre gratings [7753-361]

K. Kalli, Cyprus Univ. of Technology (Cyprus); C. Koutsides, Cyprus Univ. of Technology

(Cyprus) and Aston Univ. (United Kingdom); E. Davies, Aston Univ. (United Kingdom);

M. Komodromos, Frederick Univ. Cyprus (Cyprus); D. J. Webb, L. Zhang, Aston Univ. (United Kingdom)

$775394 \quad$ Mechanical stress sensors using micromachined grating fibers [7753-12]

H. Kumazaki, M. Hiramatsu, H. Oguri, S. Inaba, Gifu National College of Technology (Japan); K. Hane, Tohoku Univ. (Japan)

775395 Uncertainties evaluation in optical fiber grating sensor measurements [7753-106]

G. R. C. Possetti, I. Lourenço, Jr., R. C. Kamikawachi, M. Muller, J. L. Fabris, Univ. Tecnológica Federal do Paraná (Brazil)

775396 Strain sensing using long period gratings in microstructured polymer optical fibres [7753-287]

R. Lwin, A. Argyros, S. G. Leon-Saval, M. C. J. Large, The Univ. of Sydney (Australia)

775397 Fabrication and simulation of corrugated long period microfiber gratings [7753-433]

H.-Y. Wang, S.-M. Chuo, L. A. Wang, National Taiwan Univ. (Taiwan)

775398 Pressure and temperature discrimination based on dual-FBG written in microstructured fiber and standard fiber [7753-22]

C. Wu, Y. Zhang, Dalian Univ. of Technology (China); B.-O. Guan, Jinan Univ. (China)

775399 Compact fiber bending sensor based on superimposed gratings [7753-62]

Y. Liu, X. Dong, L.-Y. Shao, J. Kang, C.-L. Zhao, China Jiliang Univ. (China); C. C. Chan, Nanyang Technological Univ. (Singapore)

7753 9A Refractive index sensitivity of fibre optic long period gratings with $\mathrm{SiO}_{2}$ nanoparticle based mesoporous coatings [7753-65]

S. Korposh, S.-W. Lee, Univ. of Kitakyushu (Japan); S. W. James, R. P. Tatam, Cranfield Univ. (United Kingdom)

7753 9B Simultaneous measurement of strain and temperature by using micro-tapered long-period fiber gratings [7753-69]

M.-S. Yoon, Y.-G. Han, Hanyang Univ. (Korea, Republic of)

7753 9C Demodulation based on a long-period grating in photonic crystal fiber with differential processing for highly birefringent fiber loop mirror temperature sensor [7753-87] Y. Wang, C.-L. Zhao, X. Dong, J. Kang, S. Jin, China Jiliang Univ. (China); C. C. Chan, Nanyang Technological Univ. (Singapore)

7753 9D Novel fiber loop mirror pressure sensor using a LPG as demodulation device [7753-280] Y. Zhao, H. WU, Q. Wang, Northeastern Univ. (China) 
$77539 \mathrm{E}$ Pressure sensitivity of dual resonant long-period gratings written in boron co-doped optical fiber [7753-290]

M. Smietana, Univ. du Québec en Outaouais (Canada) and Warsaw Univ. of Technology (Poland); W. J. Bock, P. Mikulic, J. Chen, Univ. du Québec en Outaouais (Canada);

R. Wisniewski, Institute of Atomic Energy POLATOM (Poland)

$77539 \mathrm{~F} \quad$ Effective tuning of long-period grating refractive-index sensitivity by plasma-deposited diamond-like carbon nano-coatings [7753-315]

M. Smietana, Univ. du Québec en Outaouais (Canada) and Warsaw Univ. of Technology (Poland); W. J. Bock, P. Mikulic, Univ. du Québec en Outaouais (Canada); J. Szmidt, Warsaw Univ. of Technology (Poland)

$77539 \mathrm{G} \quad$ UV inscribed long period gratings with femtosecond ablated axial fibre slots for polarization control [7753-358]

E. Davies, Aston Univ. (United Kingdom); K. Kalli, C. Koutsides, Cyprus Univ. of Technology

(Cyprus); T. Allsop, L. Zhang, Aston Univ. (United Kingdom)

$77539 \mathrm{H} \quad$ Highly refractive index sensitive femtosecond laser inscribed long period gratings [7753-360]

E. Davies, Aston Univ. (United Kingdom); K. Kalli, Cyprus Univ. of Technology (Cyprus);

C. Koutsides, Aston Univ. (United Kingdom) and Cyprus Univ. of Technology (Cyprus);

L. Zhang, Aston Univ. (United Kingdom)

$775391 \quad$ Multi long-period gratings in a fiber carved and written by using a $\mathrm{CO}_{2}$ laser for distributed sensing [7753-373]

Y. Katsuyama, Y. Tokunaga, S. Kasahara, R. Sougen, O. Koyama, M. Yamada, Osaka

Prefecture Univ. (Japan)

7753 9J Temperature characteristics of microfiber long-period-gratings fabricated by a femtosecond infrared laser [7753-391]

J. Ma, W. Jin, L. Jin, The Hong Kong Polytechnic Univ. (Hong Kong, China)

7753 9K Bending sensitivity of long-period fiber gratings written in polarization-maintaining fiber by $\mathrm{CO}_{2}$ laser [7753-397]

J. Zou, Y. Liu, D. Yang, T. Wang, Shanghai Univ. (China)

$77539 \mathrm{~L}$ A spectrally and spatially multiplexed LPG sensor system using an InGaAs CCD linear array [7753-464]

P. E. Balzhiev, Technical Univ. of Sofia (Bulgaria); W. J. Bock, Univ. du Québec en Outaouais (Canada); T. A. Eftimov, Plovdiv Univ. (Bulgaria); P. Mikulic, Univ. du Québec en Outaouais (Canada); R. Arnaudov, Technical Univ. of Sofia (Bulgaria)

$77539 \mathrm{M} \quad$ Highly sensitive refractive index sensor based on two cascaded long period gratings with rotary refractive index modulation [7753-90]

T. Zhu, Y. Fan, M. Deng, Chongqing Univ. (China); Y. Rao, Univ. of Electronic Science and Technology of China (China) and Chongqing Univ. (China)

$77539 \mathrm{~N} \quad$ Long period grating for acoustic wave detection [7753-206]

J.-O. Gaudron, F. Surre, T. Sun, K. T. V. Grattan, The City Univ. (United Kingdom) 
775390 Asymmetrical twin-core fiber based long period fiber gratings [7753-269] $X$. Wang, L. Yuan, Harbin Engineering Univ. (China)

7753 9P Strain sensitivity of long period gratings in hollow-core photonic bandgap fibers [7753-296] L. Jin, TheHong Kong Polytechnic Univ. (Hong Kong, China) and Jinan Univ. (China); W. Jin, J. Ju, Hong Kong Polytechnic Univ. (Hong Kong, China)

$77539 Q \quad$ Highly sensitive operation of LPG vibration sensor using bending-induced spectral change [7753-188]

S. Tanaka, A. Wada, N. Takahashi, National Defense Academy (Japan)

7753 9R Pd-Ag film coated cascaded long period gratings for hydrogen gas sensing [7753-316] Z. Yang, Tsinghua Univ. (China); X. Yu, Heilongjiang Univ. (China); X. Chu, M. Zhang, S. Lai, Y. Liao, Tsinghua Univ. (China); Q. Li, Y. Zhang, Z. Zhuang, Institute of Structural Mechanics (China)

775395 Simultaneous measurement of temperature, hydrostatic pressure and acoustic signal using a single distributed Bragg reflector fiber laser [7753-47]

Y.-N. Tan, Y. Zhang, Dalian Univ. of Technology (China); B.-O. Guan, Jinan Univ. (China)

\section{SENSOR CHARACTERIZATION}

$77539 \mathrm{~T} \quad$ High-birefringent fiber loop mirror with an output port probe for sensing applications [7753-09]

O. Frazão, R. M. Silva, INESC Porto (Portugal); J. L. Santos, INESC Porto (Portugal) and Univ. do Porto (Portugal)

$775394 \quad$ Distributed optical fiber temperature sensor using only anti-Stokes Raman scattering light in a loop configuration [7753-384]

M. A. Soto, A. Signorini, T. Nannipieri, S. Faralli, G. Bolognini, F. Di Pasquale, Scuola Superiore Sant'Anna (Italy)

$77539 \mathrm{~V}$ Impact of Raman scattering and modulation instability on the performances of Brillouin sensors [7753-226]

S. M. Foaleng, L. Thévenaz, Ecole Polytechnique Fédérale de Lausanne (Switzerland)

7753 9W Fiber optic ultrasonic probe based on refractive-index modulation in water [7753-147] B. Shen, Y. Wada, D. Koyama, R. Isago, Y. Mizuno, K. Nakamura, Tokyo Institute of Technology (Japan)

\section{POST DEADLINE PAPER SESSION}

7753 A2 High-repetition-rate distributed Brillouin sensor by correlation domain analysis with differential frequency modulation [7753-700]

K. Y. Song, Chung-Ang Univ. (Korea, Republic of); M. Kishi, Z. He, K. Hotate, The Univ. of Tokyo (Japan)

7753 A3 Near shot-noise limited performance of an open-loop laser-driven interferometric fiber optic gyroscope [7753-701]

S. W. Lloyd, M. J. F. Digonnet, S. Fan, Edward L. Ginzton Lab., Stanford Univ. (United States) 
7753 A4 Multiplexed interferometric displacement sensing below the laser frequency noise limit [7753-702]

T. T.-Y. Lam, D. M. R. Wuchenich, J. H. Chow, D. E. McClelland, D. A. Shaddock, The Australian National Univ. (Australia)

7753 A5 Depletion in a distributed Brillouin fiber sensor: practical limitation and strategy to avoid it [7753-703]

L. Thévenaz, S. Foaleng Mafang, Ecole Polytechnique Fédérale de Lausanne (Switzerland);

J. Lin, Bucknell Univ. (United States)

7753 A6 Distributed fiber beat length, birefringence and differential group delay measurement using BOTDA technique [7753-704]

S. Xie, Univ. of Ottawa (Canada) and Tsinghua Univ. (China); X. Bao, L. Chen, Univ. of Ottawa (Canada)

7753 A7 An all polymer fibre optic sensor for measuring rapid change in oxygen partial pressure [7753-705]

R. Chen, A. D. Farmery, Univ. of Oxford (United Kingdom); A. Obeid, Oxford Optronix Ltd. (United Kingdom); C. E. W. Hahn, Univ. of Oxford (United Kingdom)

7753 A8 High-axial-resolution distributed lateral displacement measurement based on differential pulse-width pair BOTDA [7753-706]

Y. Dong, X. Bao, L. Chen, Univ. of Ottawa (Canada)

Author Index 


\title{
Conference Committee
}

\author{
Conference Chairs
}

Wojtek J. Bock, Université du Québec en Outaouais (Canada)

Jacques Albert, Carleton University (Canada)

Xiaoyi Bao, University of Ottawa (Canada)

Director of Operations

Michael G. Scott, CPC-Canadian Photonics Consortium (Canada)

Local Organizing Committee

Daniel Brabant, Université du Québec en Outaouais (Canada)

Boris Elenkrig, Ontario Centres of Excellence (Canada)

Nicole Lamoureux, OCRI-Ottawa Centre for Research and Innovation (Canada)

Kexing Liu, EcoVu (Canada)

Kathy Mahoney, OCRI-Ottawa Centre for Research and Innovation (Canada)

Mateusz Smietana, Université du Québec en Outaouais (Canada)

George Xiao, National Research Council Canada (Canada)

Jessica Zhang, Canadian Microsystems Corporation (Canada)

Andrzej Zlotorzynski, University of Ottawa (Canada)

International Steering Committee

Wojtek J. Bock, Conference Chair, Université du Québec en Outaouais (Canada)

Young J. Chung, Gwangju Institute of Science and Technology (Korea, Republic of)

Julian D. C. Jones, Herriot-Watt University (United Kingdom)

Hypolito Jose Kalinowski, Federal University of Technology (Brazil)

Wei Jin, Hong Kong Polytechnic University (Hong Kong, China)

Jose M. Lopez-Higuera, Universidad de Cantabria (Spain)

Alexis Mendez, MCH Engineering (United States)

David D. Sampson, University of Western Australia (Australia)

Glen A. Sanders, Honeywell Inc. (United States)

Nobuaki Takahashi, National Defence Academy (Japan)

Luc Thévenaz, Ecole Polytechnique Fédérale de Lausanne (Switzerland)

Marc R.-H. Voet, FOS\&S (Belgium)

Reinhardt Willsch, IPHT Jena (Germany)

Ryozo Yamauchi, Fujikura Ltd. (Japan) 
Technical Program Committee

Jacques Albert, Conference Co-Chair, Carleton University (Canada)

Ole Bang, Technical University of Denmark (Denmark)

Xiaoyi Bao, Conference Co-Chair, University of Ottawa (Canada)

John Canning, University of Sydney (Australia)

James H. Cole, Naval Research Laboratory (United States)

Stephen F. Collins, Victoria University (Australia)

Geoffrey Cranch, Naval Research Laboratory (United States)

Michel Digonnet, Stanford University (United States)

Wolfgang Ecke, IPHT Jena (Germany)

Pierre G. P. Ferdinand, CEA List (France)

Juan Hernández-Cordero, Universidad Nacional Autónoma de México (Mexico)

Jiri Homola, Institute of Photonics and Electronics of the ASCR v.v.i.

(Czech Republic)

Hisashi Izumita, Nippon Telegraph and Telephone Corporation (Japan)

Leszek R. Jaroszewicz, Military University of Technology (Poland)

Wei Jin, Hong Kong Polytechnic University (Hong Kong, China)

Byoungho Lee, Seoul National University (South Korea)

Paul Lefebvre, LxData (Canada)

Robert A. Lieberman, Intelligent Optical Systems, Inc. (United States)

Manuel Lopez-Amo, Universidad Pública de Navarra (Spain)

Cicero Martelli, Federal University of Technology, Paraná (Brazil)

Anna Grazia Mignani, Istituto di Fisica Applicata Nello Carrara (Italy)

Stephen Mihailov, Communications Research Center (Canada)

Koichiro Miyagi, Yamatake Corporation (Japan)

Yun Jiang Rao, University of Electronic Science and Technology (China)

Kentaro Nakamura, Tokyo Institute of Technology (Japan)

Kyunghwan Ken Oh, Yonsei University (South Korea)

Akira Sakamoto, Fujikura Ltd. (Japan)

José Luís Santos, INstituto de Engenharia de Sistemas e Computadores do Porto (Portugal)

Ralph Tatam, Cranfield University (United Kingdom)

Pedro Torres Trujillo, Universidad Nacional de Colombia-Sede Medellin (Colombia)

Moshe Tur, Tel Aviv University (Israel)

Johan Vlekken, FOS\&S (Belgium)

Anbo Wang, Virginia Polytechnic Institute (United States)

David Webb, University of Aston (United Kingdom)

Shinji Yamashita, University of Tokyo (Japan)

Libo Yuan, Harbin Engineering University (China)

\section{Session Chairs}

1 A Look Back at Optical Fiber Sensors

Xiaoyi Bao, University of Ottawa (Canada) 
2 Current Fiber Sensor Applications and Technologies

Jacques Albert, Carleton University (Canada)

3 Opening Plenary

Wojtek J. Bock, Université du Québec en Outaouais (Canada)

$4 \quad$ Brillouin Sensors

Moshe Tur, Tel Aviv University (Israel)

5 Micro- and Nano- Fibers

Anna Grazia Mignani, Istituto di Fisica Applicata Nello Carrara (Italy)

6 Sensor Applications

Stephen F. Collins, Victoria University (Australia)

$7 \quad$ Rayleigh Techniques and Frequency Domain Sensors

Wolfgang Ecke, IPHT Jena (Germany)

8 New Fibers

Manuel Lopez-Amo, Universidad Pública de Navarra (Spain)

9 PCF Sensors

Wei Jin, The Hong Kong Polytechnic University (Hong Kong, China)

10 FBG Sensors

John Canning, The University of Sydney (Australia)

11 Multimode Effects

Geoffrey Cranch, Naval Research Laboratory (United States)

12 Biomedical Applications

Kentaro Nakamura, Tokyo Institute of Technology (Japan)

13 Sensor Characterization

José Luís Santos, Instituto de Engenharia de Sistemas e Computadores do Porto (Portugal) 
Downloaded From: https://www.spiedigitallibrary.org/conference-proceedings-of-spie on 26 Apr 2023

Terms of Use: https://www.spiedigitallibrary.org/terms-of-use 


\title{
Introduction
}

\author{
Dear participants and readers,
}

It is a great honor and pleasure for us to publish the Proceedings of the $21^{\text {st }}$ International Conference on Optical Fiber Sensors (OFS21), on behalf of the OFS International Steering Committee (ISC), the OFS Technical Program Committee (TPC) and the OFS21 Local Organizing Committee. Since 1983 an OFS Conference has been held approximately every eighteen months, rotating between the Americas, Europe, and Asia-Pacific. This year, for the first time in history, OFS21 was held in Canada in its beautiful and friendly capital - Ottawa.

It is important to realize that OFS has never belonged to a large learned society or a professional organization; our Conference series has been able to flourish for the last 28 years thanks to a large group of dedicated volunteers around the world, to our informal structures such as the ISC and the TPC, and in a large part thanks to your continuous excellent progress in your research activities on fiber optic sensors. Being completely independent, OFS is now widely recognized around the world as a unique technical forum of the highest quality. Thanks to your excellent contributions, we are able to offer you this scientifically interesting and inspiring volume.

This volume contains extended summaries of the papers presented at the Conference, including some from two special workshops held on the Sunday preceding the official opening of the conference. The first workshop was dedicated to the history of optical fiber sensors in pioneering institutions from around the world, with presentations by three of the most senior members of our community; the second was devoted to one of the most active current applications: structural sensing, with papers by prominent researchers in companies active in the field.

As for the regular conference papers, notable trends this year include many works dealing with special fibers and coating materials to add functionality and multi-dimensional sensing modalities, sensors devices that rely on micro- and nano-structured fibers for compactness and higher sensitivities, and fiber sensors for biomedical applications, including imaging with fiber probes and fiber interferometers. Also of note is that fiber gratings remain at the heart of many optical sensor interrogation schemes and are constantly being improved by new fabrication techniques and integration with new fibers and coatings. Finally, distributed sensors are continuing to push the limits of distance and resolution, using both time and frequency domain interrogation, and to add more functionality (parameters), especially in the area of dynamic measurements. 
We hope you will enjoy reading these papers as much as we had pleasure in putting them together for you all.

\section{Wojtek J. Bock \\ Jacques Albert Xiaoyi Bao}

